\title{
Modulation instability in dispersion oscillating fibers
}

\author{
Arnaud Mussot ${ }^{1}{ }^{*}$, Matteo Conforti ${ }^{1}$, Stefano Trillo ${ }^{2}$, Francois \\ COPIE $^{1}$, AND ALEXANDRE KUDLINSKI ${ }^{1}$ \\ ${ }^{1}$ Univ. Lille, CNRS, UMR 8523 - PhLAM - Physique des Lasers Atomes et Molécules, IRCICA, F-59000 Lille, \\ France \\ ${ }^{2}$ Department of Engineering, University of Ferrara, Italy \\ *Corresponding author: arnaud.mussot@univ-lille1.fr \\ Compiled November 27, 2017
}

\begin{abstract}
In this review we present recent theoretical and experimental progresses on modulation instability and parametric amplification processes in dispersion oscillating fibers. These optical fibers are characterized by longitudinal periodic variations of their outer diameter engineered over the meter-long scale, which provides an additional degree of freedom to the system and leads to the generation of multiple MI side band pairs. Main results published in single pass configurations and in passive cavities are summarized in this review. () 2017 Optical Society of America
\end{abstract}

OCIS codes: (060.4370) Nonlinear Optic, fiber, (190.4380) Nonlinear Optics, four-wave mixing

http://dx.doi.org/10.1364/aop.XX.XXXXXX

\section{INTRODUCTION}

Modulation instability (MI) refers to the exponential amplification of a weak perturbation at the expense of a strong continuous wave [1]. It has been extensively studied in several fields of physics ranging from plasma physics [2], hydrodynamics [3], Bose-Einstein condensate [4], solid state physics [5] or nonlinear fiber optics [6]. MI is a basic nonlinear phenomenon [7] and recognized to be a key ingredient in the development of more complex nonlinear processes such as Fermi-Pasta-Ulam (FPU) recurrence [8-10], Peregrine and Kuznetsov-Ma soliton formation [11, 12], supercontinuum generation [13, 14] and rogue waves formation [15-17]. Fiber optics constitute an excellent test bed to perform experimental investigations of these fundamental phenomena since their parameters can be easily tuned by controling fiber characteristics and/or initial conditions of laser beams [11, 12, 18, 19]. In addition, from an applicative point of view, wide gain bands and large gain values offered by MI are exploited to develop fiber optical parametric amplifiers for high bit rate telecommunication applications $[20,21]$, short pulse amplification in all fiber chirped amplification systems [22], solitonic pulse train generation [23] or signal processing [24] to name a few. All these investigations has been 
achieved in uniform fibers that can limit the performances of these systems or their fundamental interest. Tajima [25] had the genuine idea to use fibers with slowly decreasing group-velocity dispersion (GVD) along the propagation axis in order to compensate for the loss experienced by solitonic pulses during their propagation in uniform fibers. The longitudinal variation of the dispersion acts as an "effective amplification" and allows deformation-free propagation of solitons in optical fibers. The first experimental demonstration has been realized by Dianov et al. [26, 27], and the concept was further developed by Chernikov and Mamyshev to achieve soliton compression in tapered fibers [28]. Later on, the idea of taking benefit of this additional degree of freedom to break the limits of uniform nonlinear fiber optics has been proposed by Kudlinski et al. [29] in the context of supercontinuum in photonic crystal fibers (PCF). They fabricated a tapered PCF to extend the low wavelength spectral edge toward the ultra-violet region, which is of primary importance in a number of biophotonics applications [30].

This additional degree of freedom thus provides a great improvement of the potential of nonlinear fiber optics, though these investigations were restricted to a monotonous longitudinal evolution of fiber parameters. It is well known in optics, that a longitudinal periodic variation of a parameter drastically modifies the light propagation behavior, conducing to the formation of forbidden photonic bandgaps [31] in linear optics or to complex phenomena such as solitary wave, discrete breathers or wave localization in nonlinear optics [32]. The length scale of these periodic structures, usually termed photonic crystals, is typically of the order of the wavelength and in practice they are fabricated via lithography processes. The advent of modern telecommunication networks with periodic all-optical regeneration devices and/or dispersion managed lines provides another field of exploration for nonlinear optics in periodic media. Length scales are in the order of kilometers, thus nonlinear phenomena that occur in these systems are fundamentally different than the ones in photonic crystals. However, a new and rich nonlinear dynamics has been discovered in these periodic systems. For instance, dispersion managed transmission lines allow for the demonstration of distortion-free soliton propagation, with soliton breathing evidence [33-36]. In the context of MI, Matera et al. [37] first predicted that the periodic variation of telecommunication network parameters leads to emergence of MI side bands, the position of which depends on the periodicity of the system $[37,38]$. For typical kilometer ranges of periodicity, MI side bands fall within the signal spectrum causing significant distortions of the latter. Consequently, a lot of investigations have been conducted in this context to provide a deeper understanding of this detrimental process for wavelength-division multiplexing optical communications [39-60]. These works reveal that MI side bands in these periodically modulated devices are ruled by a quasi-phase matching (QPM) relation and consequently, unlike MI in single mode, isotropic and uniform fibers, (i) MI can exist in the normal dispersion region and (ii) a large number of side bands can be exited. These periodic variations of fiber properties thus bring an additional degree of freedom to the system and break the conventional limitations of standard MI in uniform fibers. The first preliminary experimental observations of side bands generated via QPM have been reported in the mid 90s by Kikuchi et al. [61] in a recirculating loop with periodic variation of power, and by Murdoch et al. $[62,63]$ in fibers with periodic birefringence. However, only a weak generation of a single pair 
of side bands due to the periodicity has been reported in these works. The first clear observation of MI in a periodic fiber system has been reported in 2013 by Droques et al. [49] in a PCF with a sine modulation of its outer diameter, with a period of $10 \mathrm{~m}$. More than 10 pairs of side bands have been clearly reported experimentally by pumping in the average normal dispersion region. This observation has stimulated a renewed interest in experimental $[45,49-$ $51,53,54,56,59,60]$ as well as in theoretical [39, 42-48, 50-53, 55, 56, 58-60] investigations concerned with nonlinear optics in longitudinally modulated fibers and silicon waveguides [64].

The aim of this review is to remind the basic principles of MI in these dispersion oscillating fibers (DOFs) and to give an overview on recent experimental and theoretical achievements in these fibers. The paper is organized as follows. In section 2, we remind the basic principles of MI in dispersion oscillating fibers. The fabrication process is detailed in section 3 and a complete overview on the theory is done in section 4 . The first evidence of MI in a DOF is presented in section 5, whereas sections 6 and 7 are devoted to discuss results in the undepleted regime and parametric amplification, respectively. In section 8 , we discuss the effects related to the depleted stage of propagation. Finally, in section 9, we discuss the observation of MI in a passive cavity made of DOFs.

\section{MODULATION INSTABILITY BASIC PRINCIPLES}

In this section we present the basic properties of MI occurring in uniform and in dispersion oscillating fibers through typical examples. Let us consider the following nonlinear Schrödinger equation (NLSE) that rules MI process in optical fibers :

$$
i \frac{\partial u}{\partial z}-\frac{\beta_{2}(z)}{2} \frac{\partial^{2} u}{\partial t^{2}}+\gamma|u|^{2} u=0,
$$

With $\gamma$ the nonlinear coefficient, $\beta_{2}(z)$ the longitudinal evolution of the group velocity dispersion along the propagation axis to be of the form

$$
\beta_{2}(z)=\bar{\beta}_{2}+\beta_{M} \times \sin (2 \pi z / Z),
$$

where $Z$ is the period of modulation, $\bar{\beta}_{2}$ the average GVD and $\beta_{M}$ the amplitude of modulation. Without loss of generality, we assume that the nonlinear coefficient is constant. We will see below that it corresponds to a large variety of realistic experimental configurations. In uniform fibers $\left(\beta_{2}(z)=\right.$ constant $)$, a standard stability analysis of MI [7] reveals that perturbations at frequency $\omega$ of an intense cw pump of power $P$, can grow if the following phase matching relation is satisfied:

$$
\beta_{2} \omega^{2}+2 \gamma P=0
$$

Equation (3) implies that MI can only occur in the anomalous dispersion region of optical fibers, i.e. when $\beta_{2}<0$, in such a way that the nonlinear mismatch $\Delta k_{N L}=2 \gamma P$ exactly compensate for the GVD-induced mismatch $\Delta k=-\left|\beta_{2}\right| \omega^{2}$ of the four-photon mixing interaction between the pump and the signal-idler pair. The MI spectrum calculated at the output of the uniform fiber which dispersion is shown in Fig. 1 (a) in dashed blue curve is characterized by two phase matched symmetric side lobes whose positions are predicted by Eq. 3 (see Fig. 1 (b)). Conversely, in uniform fibers, when the dispersion is normal (red dotted lines in Fig. 1 (a)), no perfect phase matching can be achieved and thus no MI exists in these fibers (Fig. 1 (c)). 

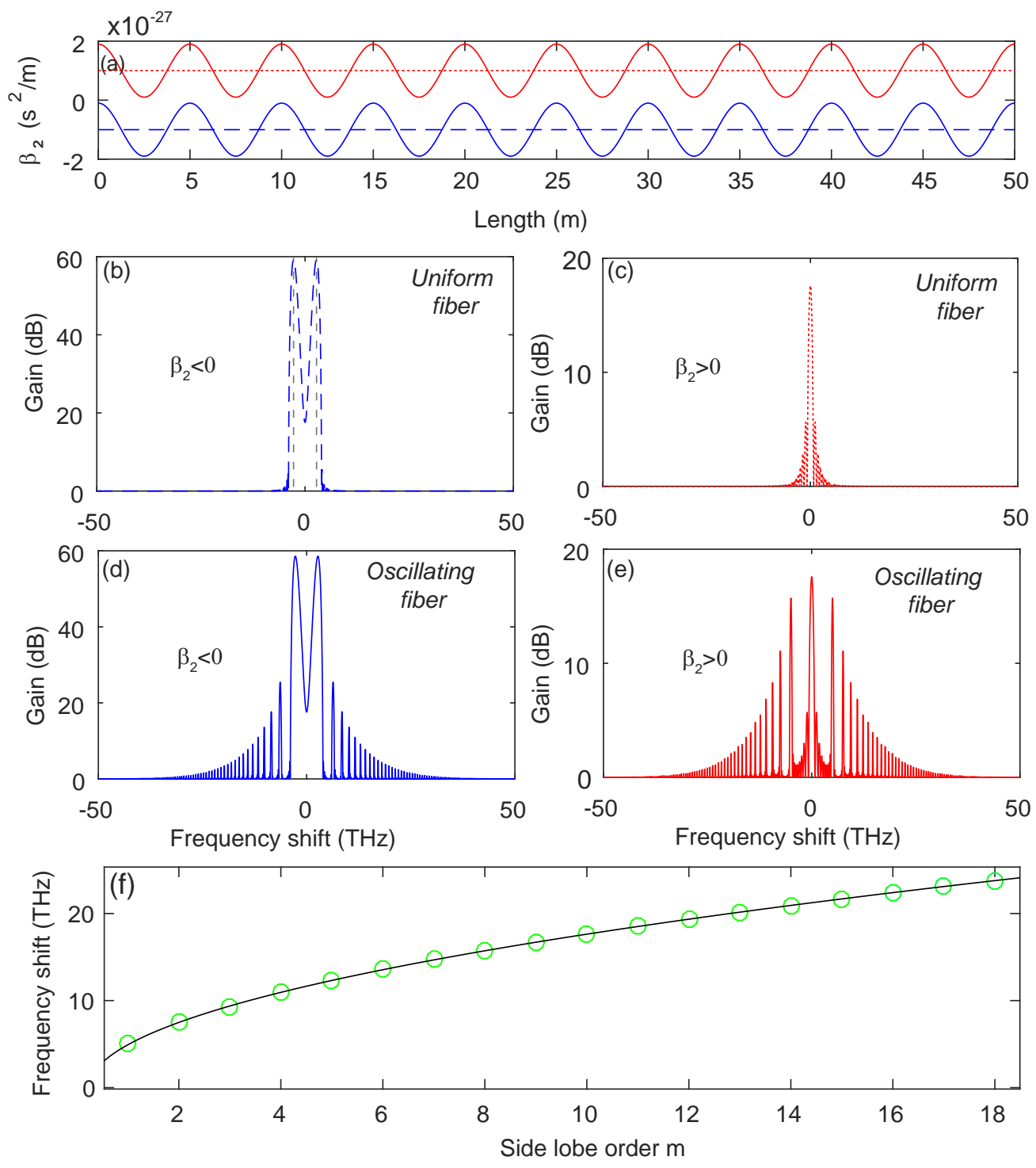

Fig. 1. (a) Group velocity dispersion evolution along the fiber. Gain spectra at the output of (b) a uniform fiber by pumping in the anomalous dispersion, (c) a uniform fiber by pumping in the normal dispersion, (d) a dispersion oscillating fiber by pumping in the anomalous dispersion and (e) a dispersion oscillating fiber by pumping in the normal dispersion. Color and line styles are the same for (a) to (e) figures. (f) detuning of the quasi-phase matched MI side lobes vs. sideband order $m$ : solid line from Eq. (4) and circles from numerical simulations shown in (e). Parameters: $\bar{\beta}_{2}= \pm 1 \times 10^{-27} \mathrm{~s}^{2} / \mathrm{m}$, $\beta_{M}=0.9 \times 10^{-27} \mathrm{~s}^{2} / \mathrm{m}, \gamma=7.5 / \mathrm{W} / \mathrm{km}, P_{P}=20 \mathrm{~W}$ and $\mathrm{Z}=5 \mathrm{~m}$. Numerical simulations are inspired form the method described in Ref. [65] where signal and idler characteristics at the output of a fiber made of several uniform piece of fibers are evaluated by means of a matrix product, each matrix depending on the uniform fiber piece characteristics. We applied this method to DOFs by sampling the fiber with a very short step to obtain a concatenation of very short uniform fiber pieces. The advantage of doing so compare to NLSE integration is that it is extremely rapid to calculate the gain curve at the output of DOFs. Note that it is possible to include linear losses and well as Raman effect as reported in Ref. [66]. We will refer to as "segmentation method" in the rest of the paper. 
The scenario changes drastically if we consider an optical fiber with a periodic modulation of its GVD. A dispersion grating associated with the periodic variation of the dispersion must be accounted for in the momentum conservation relation [37], leading to the following QPM relation:

$$
\bar{\beta}_{2} \omega^{2}+2 \gamma P=2 m \pi / Z,
$$

where $m$ is a positive or negative integer. Consequently, an infinite number of MI side bands can be destabilized in these fibers as can be seen in Fig. 1 (d) and (e) where spectra at the output of two different DOFs are represented. In both cases, the dispersion evolves sinusoidally along the fiber with the same period and amplitude of modulation but with opposite average dispersion values. Compared to uniform fibers, a set of additional side bands are generated in DOFs due to the periodic modulation of the dispersion. The frequency of these parametric sidebands can be very well estimated from the quasi-phasematching relation (Eq. 4), as can be seen in Fig. 1 (f) which represents the positions of MI side bands deduced from numerical simulations (red dots) and from theoretical predictions of the quasi-phase matching relation (Eq. 4, solid line). The limit of validity of this simple and intuitive approach is discussed in section 4 .
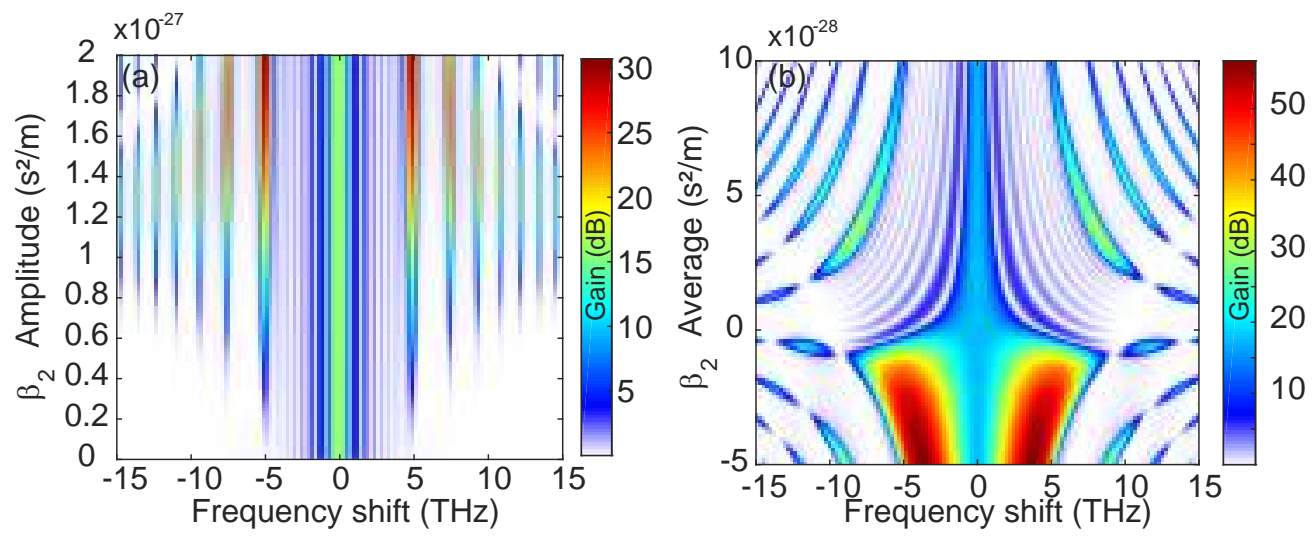

Fig. 2. 2D plots of the output gain spectrum in a dispersion oscillating fiber with a sinusoidal modulation versus, (a) the amplitude of modulation of the group velocity dispersion and (b) the average value of the group velocity dispersion. Parameters: Similar to those used in Fig.1, $\bar{\beta}_{2}$ is equal to $1 \times$ $10^{-27} \mathrm{~s}^{2} / \mathrm{m}$ in (a) and $\beta_{M}$ is equal to $0.9 \times 10^{-27} \mathrm{~s}^{2} / \mathrm{m}$ in (b).

It is important to note that the QPM relation predicts that the position of the MI side bands depends on the average dispersion value but no information related to the shape of the modulation appear in this expression. In order to illustrate this, the evolution of the gain spectrum is provided in a $2 \mathrm{D}$ plot in Fig. 2 (a) as a function of the amplitude of modulation with the same average dispersion value than the one used in Fig. 1. With no modulation, the output spectrum is similar to the spectrum shown in Fig. 2 (c) and no MI side band is excited. By increasing the modulation amplitude, symmetric side lobes start growing and their positions remain at a fixed frequency shift. Keeping on increasing the amplitude of modulation still does not affect their positions but one can see that their respective gain differ from side lobe to side lobe. This will be investigated in details in section 4 , where analytical expressions of the parametric gain will be derived. Note that in all this review, we focused 

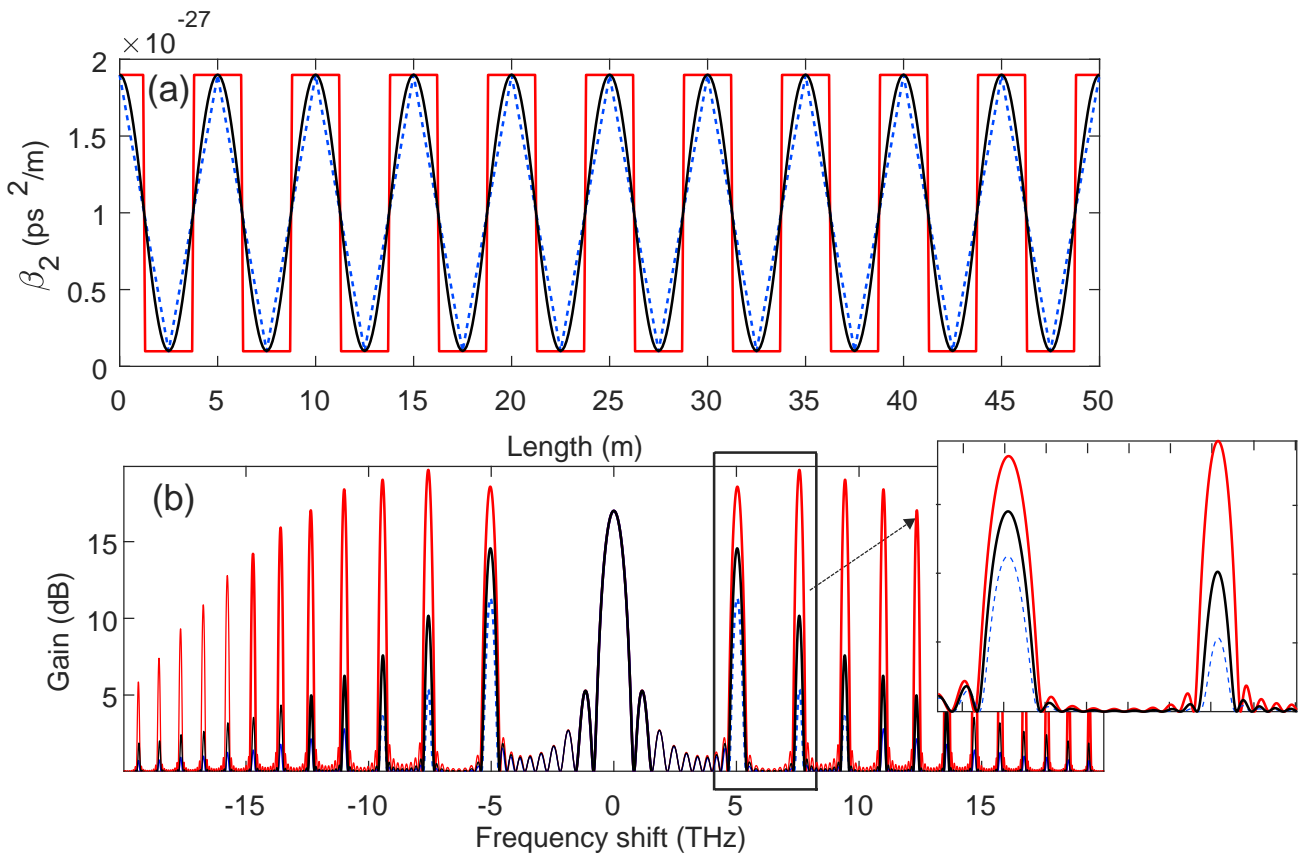

Fig. 3. (a) Group velocity dispersion evolution along the fiber length. (b) Corresponding output spectra. Inset: close-up on the first side bands. Similar parameters than those used in Fig. 1 except the modulation formats.

our attention to relatively weak amplitude of modulation, but it has been show in Ref. [55] that side band splitting might occur when the amplitude of modulation is very large.

We now illustrate the impact of the average dispersion value on the MI spectrum. Figure 2 (b) represents in a 2D plot the evolution of MI gain spectra represented as a function of the average GVD value, while keeping fixed the amplitude of modulation to $0.9 \times 10^{-27} \mathrm{~s}^{2} / \mathrm{m}$. We can clearly see that varying the average dispersion value strongly modifies the position of the side lobes as well as their gain. In anomalous dispersion, two strong symmetric side lobes corresponding to $m=0$ are located on both sides of the pump with additional ones which positions correspond to $m \neq 0$. MI side bands frequency shift increases when the absolute value of the average dispersion is decreased. In normal dispersion, only remain side bands associated to $m \neq 0$, and their respective frequency shifts are reduced by increasing the average dispersion value. The fact that the MI side band frequency shifts depend on the average dispersion value is consistent with the QPM relation (4).

Finally, the impact of the modulation format on the output spectrum is illustrated in Fig. 3, where MI spectra obtained in DOFs with different modulation formats are shown. We choose a sine, a square and a triangle (Fig. 3 (a)) modulation formats with the same average dispersion value. All other parameters are similar to those used in Fig. 1. Firstly, positions of the side bands are almost equal for all these modulation formats (Fig. 3 (b)). Again, it simply illustrates that the position of the MI side bands depends on the average dispersion value. Secondly, the amplitude of MI orders strongly depends on the shape of the modulation. For instance, the gain experienced by the first side band is $19 \mathrm{~dB}$ for the square, $15 \mathrm{~dB}$ for the sine and $12 \mathrm{~dB}$ for the triangle modulation shape. 
More generally, gain values for each side lobes are different for all MI orders. This simple example illustrates that the position of MI side bands depends on the average dispersion value while their respective gains also depend on the shape of the modulation. This will be investigated in details in section 4 .

\section{FABRICATION OF DISPERSION OSCILLATING FIBERS}

Most experimental results presented in this manuscript have been achieved in PCFs because they allow a much greater control over dispersion than conventional fibers. However, the fabrication of DOFs from standard step-index telecommunication fiber preforms is of course possible too. It has been reported in dispersion managed systems for telecommunication applications in 2003 [67] or in the context of soliton collision in 2007 [68]. Note that, in the context of MI, DOFs made from standard telecommunication fibers have been used by us [54] and by Finot and coworkers [60]. Since most experimental results related to MI have been carried out with modulated PCFs, we remind here the basic principle of fabrication of PCFs, by emphasizing how the modulation of their diameter affects their guiding properties.

\section{A. Fabrication principles}

We use the standard stack and draw technique [69] to fabricate the photonic crystal fibers (PCFs). The stacked array of capillaries is drawn into canes of a few $\mathrm{mm}$ diameter, which are then jacked in pure silica tubes to form the final preform, which is drawn into fibers. The air holes of the PCF are pressurized to prevent their collapse during the drawing process. When fabricating uniform fibers with a targeted outer diameter $d$, the drawing speed is usually adjusted by a feedback loop in quasi real time in order to maintain the fiber diameter as close as possible to $d$. The typical accuracy over $d$ is in the order of $\pm 1 \%$. For the fabrication of periodically modulated fibers, the longitudinal evolution of the fiber diameter is controlled by adjusting the evolution of drawing speed with time (which is related to fiber length) using a servo-control system. This process is ruled by the conservation of glass mass between the preform (jacketed cane) and the fiber:

$$
d_{\text {Fiber }}=d_{\text {Preform }} \sqrt{\frac{V_{\text {Preform }}}{V_{\text {Fiber }}}}
$$

where $d_{\text {Preform,Fiber }}$ are respectively the preform (jacketed cane) and fiber outer diameter, and $V_{\text {Preform,Fiber }}$ are respectively the preform feed into the furnace and the drawing capstan speed. In our process, $d_{\text {Preform }}$ and $V_{\text {Preform }}$ are fixed, $V_{\text {Fiber }}$ is adjusted with a desired $f(z)$ (where $z$ is the longitudinal space coordinate along the fiber) function, which results in a modulation of the fiber outer diameter $d_{\text {Fiber }}(z)$, and thus of the overall photonic crystal structure. By suitably adjusting the fiber tension and hole pressure during drawing, the normalized air hole diameter $d / \Lambda$ (where $\Lambda$ is the pitch of the microstructure) can be kept constant all along the periodically modulated PCF. This means that the pitch value (and the core diameter for instance) follow a linear dependence with outer diameter. This results in a periodic modulation of the mode(s) propagation constant(s), and thus of all guiding properties.

\section{B. Typical example}

The impact of the longitudinal modulations of the outer diameter on the modes propagation constants of a typical air silica PCF is illustrated in Figs. 4. The 

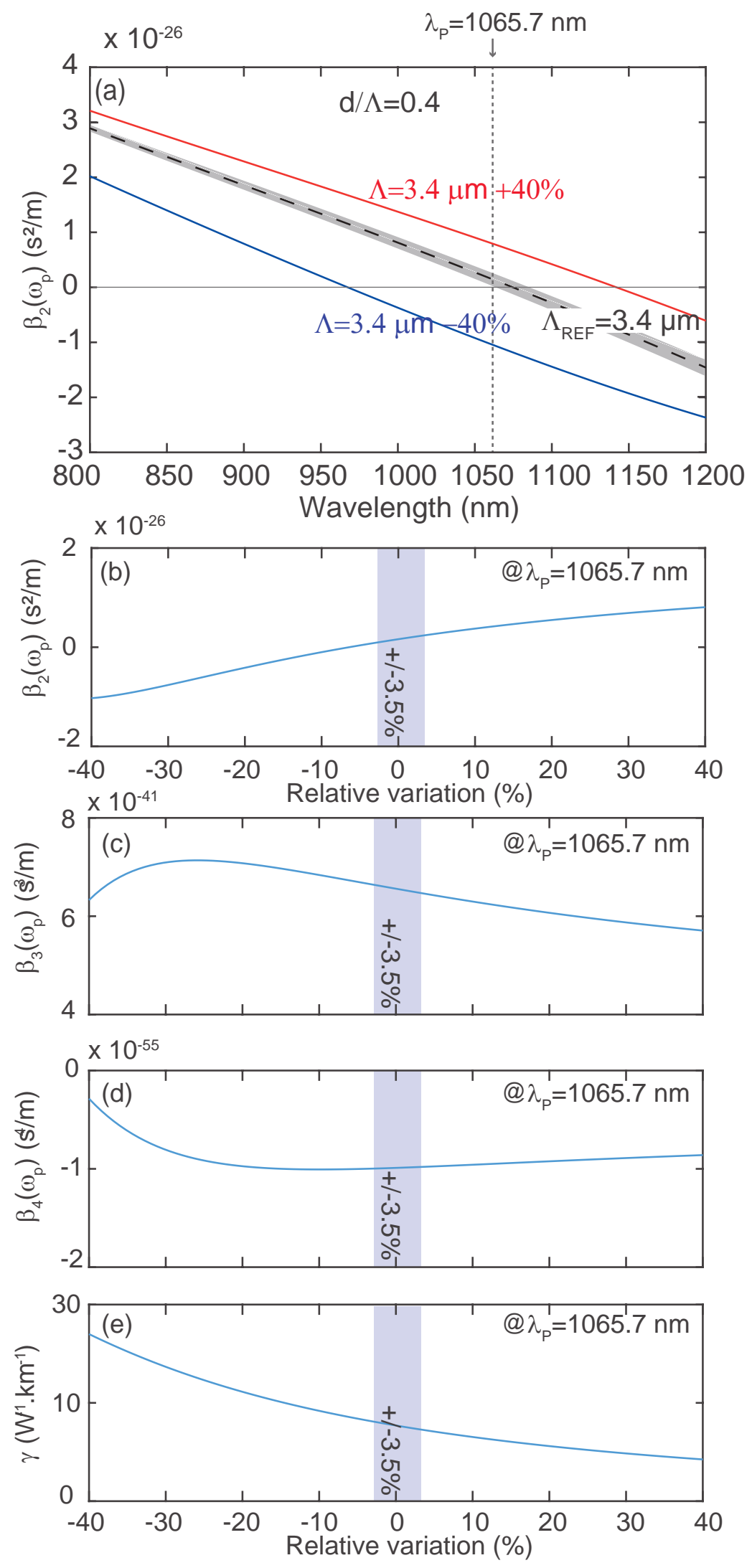

Fig. 4. (a) GVD evolution versus wavelength for different pitch values, $\Lambda_{R E F}=3.4 \mu \mathrm{m}$ (dashed black lines), $\Lambda_{M A X}=4.76 \mu \mathrm{m}$ (solid blue line) and $\Lambda_{\min }=2.04 \mu \mathrm{m}$ (solid red line). The grey area delimits a variation of $\pm 7 \%$ of the pitch around the reference value. (b)-(e) Evolutions of $\beta_{2,3,4}$ and $\gamma$ versus the normalized value of the pitch $\left[\left(\Lambda-\Lambda_{R E F}\right) / \Lambda_{R E F}\right]$. 
$d / \Lambda$ ratio is fixed to 0.4 to get an endlessly single mode behaviour [70]. Figure
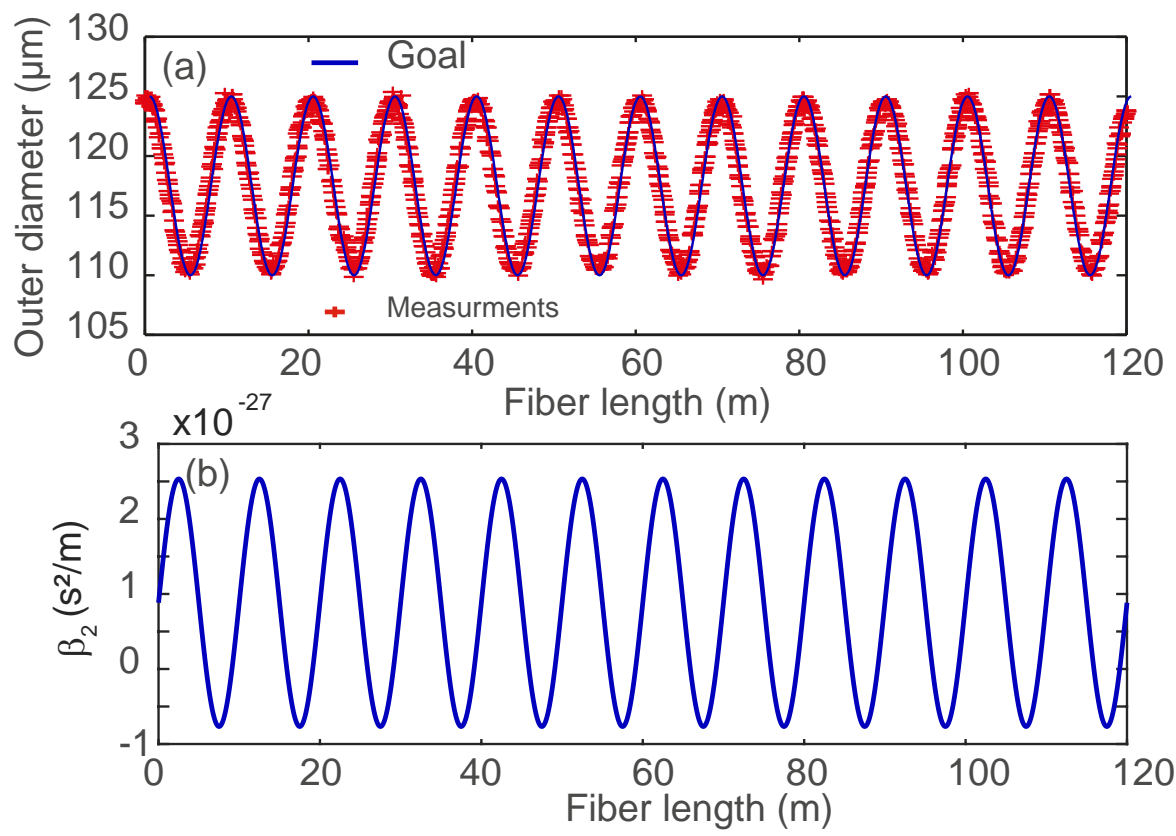

Fig. 5. (a) Outer diameter evolution with $\pm 7 \%$ variations, targeted curve (solid line), measurements (crosses). (b) Calculated GVD at $1065.7 \mathrm{~nm}$.

4(a) represents the evolution of the GVD versus wavelength for different pitch values calculated from semi-analytical relations derived in Ref. [71]. We define a reference value for the pitch, labeled $\Lambda_{R E F}$, equals to $3.4 \mu \mathrm{m}$. In the following, modulation amplitudes will be referred to this value. For $\Lambda_{R E F}$, the zero dispersion wavelength (ZDW) is located at $1074 \mathrm{~nm}$ (dashed black curve in Fig.4(a)). By decreasing the pitch value to $2.04 \mu \mathrm{m}(-40 \%)$, the curve is red shifted (solid red curve) and the ZDW is located at $1153 \mathrm{~nm}$. On the contrary, by increasing the pitch value to $4.76 \mu \mathrm{m}(+40 \%)$ the curve experiences a blue shift (solid blue curve) and the ZDW is located at $957 \mathrm{~nm}$. Consequently, by varying the outer diameter of an air silica PCF of only a few tens of percent one can induce very large wavelength shift of the GVD curve (more than $200 \mathrm{~nm}$ on the $\mathrm{ZDW}$ in this case). In order to give a clearer insight of these variations, Fig. 4 (b) to (e) represents the evolution of $\beta_{2,3,4}$ and $\gamma$, respectively, as a function of the relative variation of the pitch $\left(\left(\Lambda-\Lambda_{R E F}\right) / \Lambda_{R E F}\right)$. All these parameters are calculated at $1065.7 \mathrm{~nm}$. The GVD experiences the largest variations compared to other parameters since it moves from negative to positive values. As can be seen on these figures, these parameters are not linearly dependent on the pitch of the fiber over such large variations. However, limiting the amplitude of variation to $\pm 7 \%$ of $\Lambda_{R E F}$, we can reasonably consider that $\beta_{2}$ is proportional to the pitch variations. In addition, other parameters can be assumed to be constant over this variation span. Indeed, we checked numerically through many realistic examples that this assumption is valid in most configurations. This strongly simplifies the fabrication of dispersion oscillating fibers as the shape of the desired longitudinal variations of one physical parameter is similar to the outer diameter one (the control parameter in our drawing tower). As an example, the evolution of the GVD at $1065.7 \mathrm{~nm}$ of an optical fiber which outer diameter is sinusoidally modulated with an amplitude of $\pm 7 \%$ is represented in 
Figs. 5. The period of modulation is $Z=10 \mathrm{~m}$ and we can see that the GVD also experiences a quasi-sinusoidal evolution. Note that the example depicted in Fig. 1 (a) and (e) would correspond to a fiber with an outer diameter variation of $\pm 3.5 \%$ and by working at $1065.7 \mathrm{~nm}$. Before performing experiments in

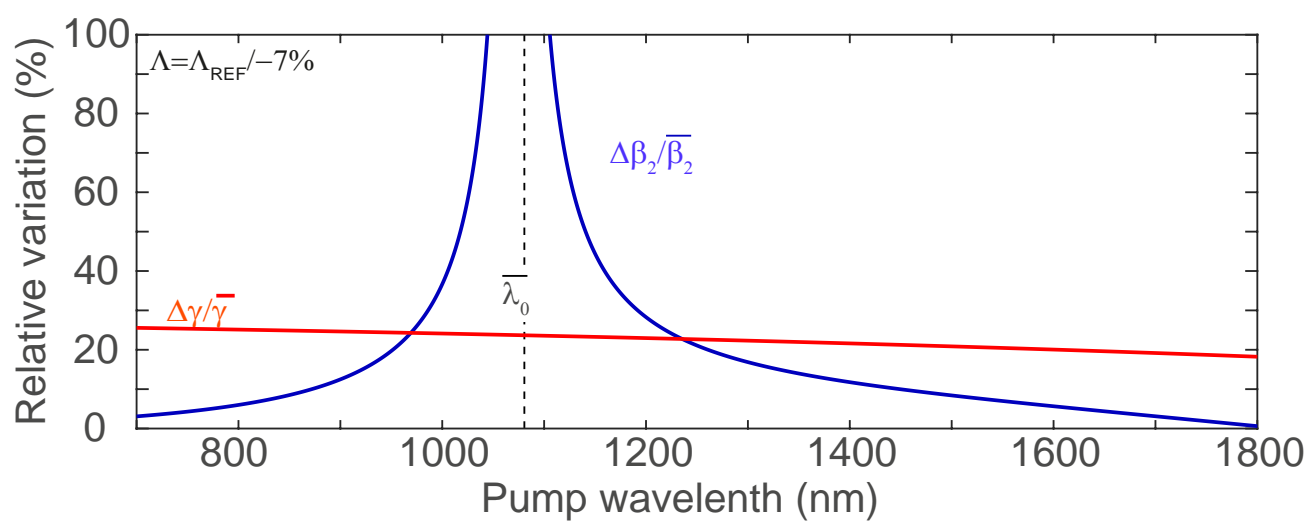

Fig. 6. Relative variation of the GVD $\left[\frac{\beta_{2}^{\operatorname{Max}}-\beta_{2}^{\min }}{\bar{\beta}_{2}}\right]$ and of the nonlinear coefficient $\left[\frac{\gamma^{\operatorname{Max}}-\gamma^{\min }}{\bar{\gamma}}\right]$ as a function of the pump.

these fibers, one question is : what is the simplest model to use to account for nonlinear effects in such periodically modulated systems? It would be possible to account for all the longitudinal variations in the NLSE equation, however, this would lead to a complex equation useless to explain the dynamics of nonlinear processes. In order to identify the simplest equation, we have to establish if the modulation amplitude of a parameter is dominant with regards to other ones. To do so, the relative variations of $\beta_{2}$ are compared to those of $\gamma$ as a function of the wavelength in Fig. 6 for very small variations of the pitch of $\pm 7 \%$ around the reference value of the pitch $\left(\Lambda_{R E F}=3.4 \mu \mathrm{m}\right)$. As it can be seen, the relative variation of the nonlinear coefficient is almost constant while the wavelength is varied from $700 \mathrm{~nm}$ to $1800 \mathrm{~nm}$. On the contrary, the relative variation of $\beta_{2}$ experiences large fluctuations on this wavelength span. It tends toward infinity close to the average ZDW $\left(\bar{\lambda}_{0}\right)$ of the fiber and approaches zero for large wavelength shifts. This makes an important difference to model the behavior of a wave propagation in such fibers. We checked numerically that close to the average ZDW, the GVD variations dominate and one can consider that the nonlinear coefficient is constant. It leads to this simplified NLSE equation, which accurately reproduces the dynamics of the system :

$$
i \frac{\partial u}{\partial z}-\frac{\beta_{2}(z)}{2} \frac{\partial^{2} u}{\partial t^{2}}+\gamma|u|^{2} u \simeq 0
$$

On the contrary, working far away from the average ZDW of the fiber is equivalent to considering that the contribution of the longitudinal variations of the nonlinear coefficient are dominant compare to those of the dispersion. The simplified NLSE equation thus reads:

$$
i \frac{\partial u}{\partial z}-\frac{\beta_{2}}{2} \frac{\partial^{2} u}{\partial t^{2}}+\gamma(z)|u|^{2} u \simeq 0
$$

In all the experimental results presented in this article, we will always work in the vicinity of the average ZDW of the fiber to get very large variation of 
the GVD while keeping relatively small outer diameter variations. In that way it is possible to exalt the contribution of the periodic variations on nonlinear processes while strongly relaxing fabrication constraints. Consequently, the simplest governing equation to accuracy model nonlinear phenomena in DOFs is then Eq. (6).

\section{THEORETICAL FRAMEWORK}

\section{Floquet analysis}

In this section we develop a general theory that permits to identify the frequency bands that can be destabilized by a periodic modulation of any of its parameters the fiber dispersion or the nonlinearity. Let us consider the following NLSE

$$
i \frac{\partial u}{\partial z}-\frac{\beta_{2}(z)}{2} \frac{\partial^{2} u}{\partial t^{2}}+\gamma(z)|u|^{2} u=0
$$

where we assume the $z$-dependent GVD $\beta_{2}(z)$ and the nonlinear coefficient $\gamma(z)$ to be of the form

$$
\beta_{2}(z)=\bar{\beta}_{2}+\beta_{M} f_{Z}(z), \quad \gamma(z)=\bar{\gamma}+\gamma_{M} g_{Z}(z),
$$

where $f_{Z}$ and $g_{Z}$ are periodic functions of period $Z$ such that $\min f_{Z}=-1=$ $\min g_{Z}$, and their mean is vanishing, i.e. $\int_{-Z / 2}^{Z / 2} f_{Z}(z) d z=\int_{-Z / 2}^{Z / 2} g_{Z}(z) d z=$ 0 . A representative example of such kind of modulation function is represented by $f_{Z}=\sin \left(k_{g} z\right)=\sin (2 \pi / Z \cdot z)$. Our aim is to analyse the stability of the general stationary (or CW) solution of Eq. (8) which reads $u_{0}(z)=\sqrt{P} \exp \left(i P \int_{0}^{z} \gamma\left(z^{\prime}\right) z^{\prime}\right)$, where $P$ is the power.

We consider a perturbation of $u_{0}(z)$ in the form $u(z, t)=[v(z, t)+1] u_{0}(z)$, where the perturbation $v(z, t)$ satisfies $|v| \ll 1$. Inserting this expression into Eq. Eq. (8), and retaining only the linear terms, we find

$$
i \frac{\partial v}{\partial z}-\frac{\beta_{2}(z)}{2} \frac{\partial^{2} v}{\partial t^{2}}+\gamma(z) P\left(v+v^{*}\right)=0
$$

Writing $v=q+i p$, with $q$ and $p$ real functions, we obtain the following linear system:

$$
\left\{\begin{array}{l}
\frac{\partial q}{\partial z}-\frac{\beta_{2}(z)}{2} \frac{\partial^{2} p}{\partial t^{2}}=0 \\
\frac{\partial p}{\partial z}+\frac{\beta_{2}(z)}{2} \frac{\partial^{2} q}{\partial t^{2}}-2 \gamma(z) P q=0
\end{array}\right.
$$

Finally, taking the Fourier transform of this system in the time variable $t$, leads to

$$
\left\{\begin{array}{l}
\frac{\partial \hat{q}}{\partial z}+\frac{\beta_{2}(z)}{2} \omega^{2} \hat{p}=0 \\
\frac{\partial \hat{p}}{\partial z}-\frac{\beta_{2}(z)}{2} \omega^{2} \hat{q}-2 \gamma(z) P \hat{q}=0 .
\end{array}\right.
$$

Note that this is a Hamiltonian dynamical system in a two-dimensional phase plane with canonical coordinates $(\hat{q}, \hat{p})$. Analyzing the linear stability of the stationary solution $u_{0}(z)$ therefore reduces to studying the solutions to Eq. (11) for each $\omega$. Since the coefficients in the equation are $z$-periodic with period $Z$, Floquet theory applies $[39,51]$. This amounts to study the linearized evolution over one period $Z$, to obtain the Floquet map $\Phi$ which in the present situation is 
the two by two real matrix defined by $\Phi[\hat{q}(0), \hat{p}(0)]^{T}=[\hat{q}(Z), \hat{p}(Z)]^{T}$. In other words, the Floquet map is given by the fundamental matrix solution of system Eq. (11) evaluated at $z=Z$. As a result $[\hat{q}(n Z), \hat{p}(n Z)]^{T}=\Phi^{n}[\hat{q}(0), \hat{p}(0)]^{T}$. Note that $\Phi$ necessarily has determinant one, since it is obtained by integrating a Hamiltonian dynamics, which preserves phase space volume. As a consequence, if $\lambda$ is one of its eigenvalues, then so are both its complex conjugate $\lambda^{*}$ and its inverse $\lambda^{-1}$. This constrains the two eigenvalues of $\Phi$ considerably: they are either both real, or lie both on the unit circle. Now, the dynamics is unstable only if there is one eigenvalue $\lambda$ satisfying $|\lambda|>1$, in which case both eigenvalues are real. We will denote as $\lambda^{ \pm}$the two eigenvalues of $\Phi$. We are interested in studying the growth rate, or parametric gain, that is defined as

$$
g(\omega)=\frac{1}{Z} \ln \left(\max \left\{\left|\lambda^{+}\right|,\left|\lambda^{-}\right|\right\}\right)
$$

as a function of $\omega, \beta_{M}$ and $\gamma_{M}$. The gain $g$ measures the growth of the perturbations $(\hat{q}(z), \hat{p}(z)) \propto \exp [g(\omega) z]$. It vanishes if the two eigenvalues lie on the unit circle. The regions where the gain does not vanish are commonly referred to as Arnold tongues [46]. We will explain below that, whereas their precise form depends on the choice of $f_{Z}, g_{Z}$, the position of their tips does not.

Since the system Eq. (11) is not autonomous, it cannot be solved analytically in general. Specific cases where analytical solutions can be found is piece-wise constant [39] and delta kicked [51] dispersion profiles. Nevertheless, the above observations will allow us to obtain some information about its stability for small $\beta_{M}, \gamma_{M}$, and valid for all perturbations $f_{Z}, g_{Z}$, whatever their specific form.

To see this, we first consider the case $\beta_{M}=0=\gamma_{M}$, so that $\beta(z)=\bar{\beta}_{2}$, $\gamma(z)=\bar{\gamma}$. It is then straightforward to integrate the system Eq. (11). The Floquet map is then given by

$$
\Phi_{a v}=\left(\begin{array}{cc}
\cos \left(k_{a v} Z\right) & -\frac{\bar{\beta}_{2} \omega^{2}}{2 k_{a v}} \sin \left(k_{a v} Z\right) \\
\frac{2 k}{\bar{\beta}_{2} \omega^{2}} \sin \left(k_{a v} Z\right) & \cos \left(k_{a v} Z\right)
\end{array}\right),
$$

where

$$
k_{a v}=\sqrt{\frac{\bar{\beta}_{2}}{2} \omega^{2}\left(\frac{\bar{\beta}_{2}}{2} \omega^{2}+2 \bar{\gamma} P\right)} .
$$

We concentrate on the normal average dispersion (defocusing) regime $\bar{\beta}_{2}>0$, since we want to characterize instabilities generated by the periodicity. The anomalous dispersion (focusing) regime is unstable even in homogeneous fibers through the standard MI. Note that the matrix $\Phi_{a v}$ has determinant equal to 1 , as expected. The eigenvalues of $\Phi_{a v}$ can be readily computed as

$$
\lambda_{a v}^{ \pm}=\exp \left( \pm i k_{a v} Z\right)
$$

If we now consider the fiber modulation terms $f_{Z}(z)$ and $g_{Z}(z)$, it is then no longer possible, in general, to give a simple closed form expression of the solution to the system Eq. (11), which is no longer autonomous, and hence of the Floquet map $\Phi$. Nevertheless, we do know that, for small $\beta_{M}, \gamma_{M}$, the eigenvalues of $\Phi$ must be close to the eigenvalues $\lambda_{a v}^{ \pm}$. We then have two cases to consider. 


$$
k \neq \frac{m \pi}{Z}
$$

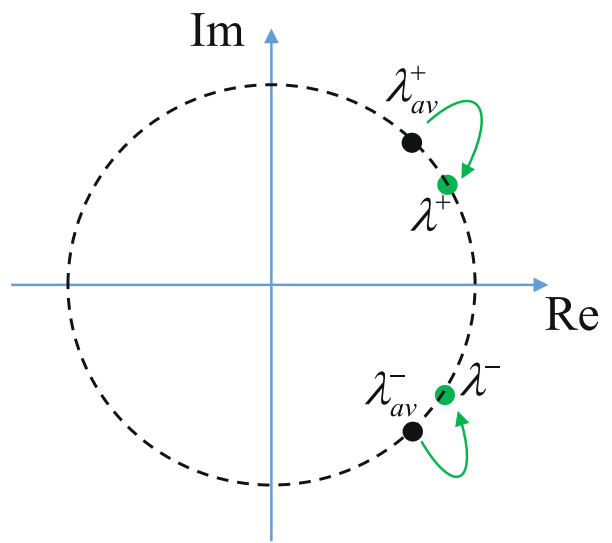

$$
k=\frac{m \pi}{Z}
$$

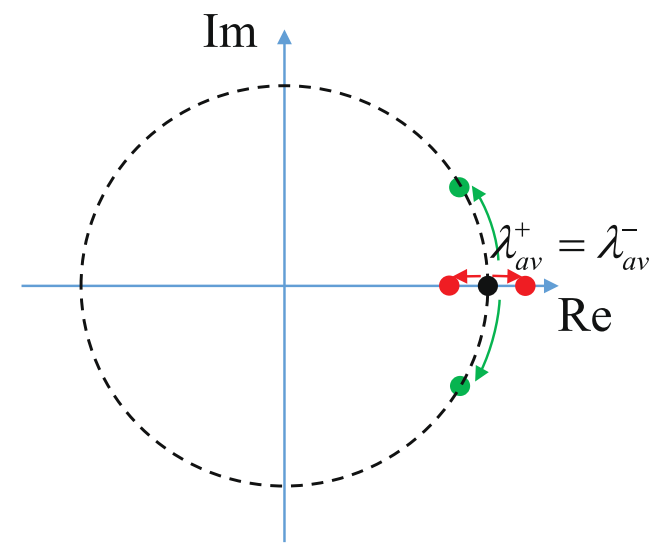

Fig. 7. Sketch illustrating, in the complex plane, the effect of the periodic modulation terms $f_{Z}(z)$ and $g_{Z}(z)$ on the eigenvalues of the linearized Floquet map (13). Black dots correspond to the unperturbed eigenvalues lying on the unit circle (dashed line). Coloured dots show the new position of the eigenvalues after switching on the modulations, leading to a stable regime when $k \neq \frac{\pi m}{Z}$ (left sketch) and an unstable one when $k=\frac{\pi m}{Z}$ (right sketch, red dots).

1: Off-resonance case. $k_{a v} \neq \frac{\pi m}{Z}, m= \pm 1, \pm 2, \ldots$. We have $\lambda_{a v}^{-}=\left(\lambda_{a v}^{+}\right)^{*}$, they are distinct, and they both lie on the unit circle, away from the real axis. They then must remain on the unit circle under perturbation since, for the reasons explained above, they cannot move into the complex plane away from the unit circle. Consequently, in this case, the stationary solution $u_{0}(z)$ is linearly stable under a sufficiently small perturbation described by $\beta_{M} f_{Z}(z)$ and $\gamma_{M} g_{Z}(z)$, and this statement does not depend on the precise form of $f_{Z}(z)$ or of $g_{Z}(z)$. In fact, with growing $\beta_{M}$ and /or $\gamma_{M}$, the two eigenvalues will move along the unit circle until they meet either at -1 or at +1 for some critical value of the perturbation parameters. Only for values of the latter above that critical value can the system become unstable. A pictorial description of this situation is shown in the panel of Fig. 7.

2: On-resonance case. $k_{a v}=\frac{\pi m}{Z}, m= \pm 1, \pm 2, \ldots$. Now $\lambda_{a v}^{+}=\lambda_{a v}^{-}= \pm 1$ (upper or lower sign holds for $m$ even or odd, respectively) is a doubly degenerate eigenvalue of $\Phi_{a v}$. Under a small perturbation, the degeneracy can be lifted and two real eigenvalues can be created, one greater than one, one less than one in absolute value, implying the instability of the system. In principle, under very peculiar perturbations, the eigenvalues might also move along the circle implying that the system remain stable. However, for the most common perturbations (sinusoidal, square wave, sawtooth, comb, ...), the system, for some $m$, destabilizes under an arbitrarily small perturbation, following the split of the eigenvalues on the real axis at the degenerate points \pm 1 . A pictorial description of this situation is shown in the right panel of Fig. 7.

We recap by saying that, when the modulation is switched on, the instability sets in under the resonant condition $k_{a v}=\frac{m \pi}{Z}$. Recalling the expression of $k_{a v}=k_{a v}(\omega)$ in Eq. Eq. (14), it is straightforward to show that the $m$-th order 
resonance is fulfilled at frequency $\omega=\omega_{m}$, where

$$
\omega_{m}=\sqrt{\frac{2}{\bar{\beta}_{2}}\left(\sqrt{(\bar{\gamma} P)^{2}+\left(\frac{m \pi}{Z}\right)^{2}}-\bar{\gamma} P\right)},
$$

which is therefore the frequency at which the system destabilizes for an infinitely small Hamiltonian perturbation of $\Phi_{a v}$. These values $\omega_{m}$ therefore correspond to the tips of the Arnold tongues, that is, to the positions of the (centers of) the unstable sidebands of the defocusing NLSE under a general periodic perturbation $f_{Z}, g_{Z}$.

We emphasize that the resonance condition

$$
k_{a v}\left(\omega_{m}\right)=m \frac{\pi}{Z}=m \frac{k_{g}}{2}
$$

is the condition of parametric resonance, i.e. the natural spatial frequency of the unperturbed harmonic oscillator $\left(k_{a v}\right)$ is equal to a multiple of half the forcing spatial frequency $\left(k_{g} / 2=\pi / Z\right)$ [46]. Additional physical insight can be obtained by expanding Eq. (16) for small power, i.e. assuming $\bar{\gamma} P \ll|m| \pi / Z$. This assumption is valid in a large range of experimental setups which period of modulation lies in the meter range and with pump power up to tens Watt. At zero order we recover the well known quasi-phase-matching relation (cfr. Eq. (4)) $[37,38,49]$ :

$$
\bar{\beta}_{2} \omega_{m}^{2}+2 \bar{\gamma} P=\frac{2 \pi m}{Z} .
$$

Equation (18) entails the conservation of the momentum (corrected for nonlinear phase-shifts), made possible thanks to the virtual momentum carried by the dispersion grating, of the four-photon mixing interaction between two photons from the pump, going into two photons in the symmetric unstable bands at lower (Stokes) and higher (antiStokes) frequencies with respect to the pump.

An example of practical interest where the Floquet analysis can be performed analytically is a fiber with a piecewise constant dispersion and uniform nonlinearity $\gamma(z)=\bar{\gamma}=\gamma$. This case was studied also in the context of communication system with dispersion management [39]. The Floquet map is given simply by the product of the two matrices describing each uniform segment:

$$
\Psi=\Phi_{a} \Phi_{b},
$$

where $\Phi_{a, b}$ has the expression Eq. (13) calculated for a dispersion $\beta(z)=\beta_{a, b}$, where the two pieces of fiber has length $L_{a, b}$, such that $L_{a}+L_{b}=Z$ and the average dispersion is $\bar{\beta}_{2}=\left(\beta_{a} L_{a}+\beta_{b} L_{b}\right) / Z$.

The eigenvalues of $\Psi$ are given by

$$
\lambda^{ \pm}=\frac{\Delta}{2} \pm \sqrt{\frac{\Delta^{2}}{4}-1},
$$

where

$$
\Delta=2 \cos \left(k_{a} L_{a}\right) \cos \left(k_{b} L_{b}\right)-\sigma \sin \left(k_{a} L_{a}\right) \sin \left(k_{b} L_{b}\right),
$$

is the Floquet discriminant, and $\sigma=\left(\beta_{a} \beta_{b} \omega^{4}+2 \gamma P \omega^{2}\left(\beta_{a}+\beta_{b}\right)\right) /\left(2 k_{a} k_{b}\right)$. We have parametric instability if $|\Delta|>2$, with gain $g(\omega)=\ln \left(\max \left|\lambda^{ \pm}\right|\right) / Z$.

In Fig. 8 we report an example of analytically calculated instability gain. We can clearly see generation of several branches (only the first three are shown), 

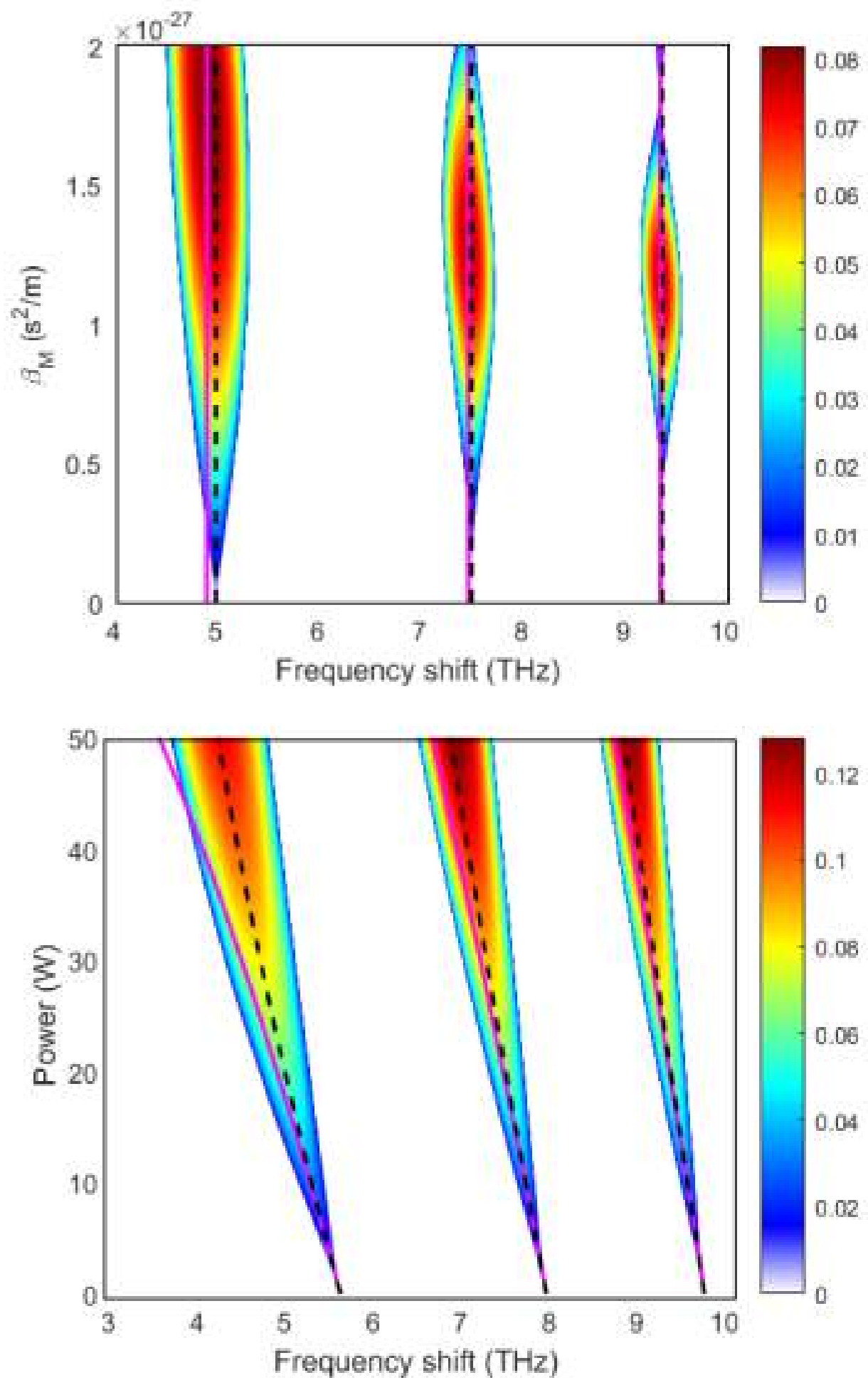

Fig. 8. Color level plot of the parametric $g(\omega)$ for a fiber with periodic GVD modulation $\bar{\beta}_{2}=1 \times 10^{-27} \mathrm{~s}^{2} / \mathrm{m}, \beta_{a, b}=1 \pm \beta_{M}, L_{a}=L_{b}=\mathrm{Z} / 2=2.5 \mathrm{~m}$, $\gamma=7.5 / \mathrm{W} / \mathrm{km} . P=20 \mathrm{~W}$ for (a) and $\beta_{M}=1 \times 0.9^{-27} \mathrm{~s}^{2} / \mathrm{m}$. Dashed black curves indicate the parametric resonance frequencies from Eq. (16), whereas magenta curves indicate the quasi phase matching frequencies from Eq. (4). 
the Arnold tongues, due to the periodic forcing. Figure 8 (a) shows the gain as a function of frequency and the amplitude of GVD modulation for a power of $20 \mathrm{~W}$ (same parameters as in Fig. 2 (a)). The position of the unstable bands is perfectly predicted by Eq. (16) (dashed black curves). In this case, the simpler QPM formula Eq. (4) (magenta curves). Figure 8 (b) shows the gain as a function of frequency and pump power for a GVD modulation $\beta_{M}=1 \times 0.9^{-27} \mathrm{~s}^{2} / \mathrm{m}$. For high enough power $(>20 \mathrm{~W}) \mathrm{Eq}$. (4) fails to accurately predict the frequency of the peak gain.

\section{Truncated three wave model}

The Floquet linear stability analysis presented in the previous section gives analytical results for some special modulation formats, namely piecewise constant $[38,72]$ and delta combs [51]. Such an analysis allows also to find the spectral positions of the parametric gain bands whatever the modulation format. However, it does not provide any clear insight into the dynamics of the process nor any details about the fine evolution of the field over a single modulation period of the fiber. To this aim, some of us proposed in Ref. [45] a more intuitive explanation of the results from Ref. [49] by revisiting a simplified truncated three-wave model usually aimed at describing FPU recurrence and fiber-optic parametric amplification [9,73-75]. This model allows to account for the relative phase variations between pump, signal and idler waves during propagation. In Ref. [45], we included a sinusoidal modulation of dispersion. Our starting point is the three truncated wave model Ref. [73-75]. We neglect fiber loss, we assume that the pump remains undepleted and that signal and idler powers, $P_{s}$ and $P_{i}$, are much weaker than the pump power $P_{p}$ over the whole DOF length. After lengthily but straightforward calculations, we obtained the following expression for local linear gain [45]:

$$
g(\omega, z)=2 \gamma P_{p} \sum_{q=-\infty}^{q=+\infty} J_{q}\left(\frac{\beta_{M} \omega^{2}}{2 \pi / Z}\right) \sin \left[\left(\overline{\beta_{2}} \omega^{2}+2 \gamma P_{p}-\frac{q 2 \pi}{Z}\right) z+K_{q}\right]
$$

with $K_{q}=\frac{\beta_{M} \omega^{2}}{2 \pi / Z}-q \frac{\pi}{2}+\theta(\omega, 0)$ and the following relation for the relative phase $\theta(\omega, z)$ :

$$
\theta(\omega, z)=\left[\overline{\beta_{2}} \omega^{2}+2 \gamma P_{p}\right] z+\frac{\beta_{M} \omega^{2}}{2 \pi / Z}[1-\cos (2 \pi z / Z)]+\theta(\omega, 0)
$$

Thus, Eq. 22 indicates that the linear gain $g(\omega, z)$ at a fixed pulsation detuning $\omega$ can be interpreted as the sum of sine functions in $z$. These sine functions all have a zero average value except when their argument becomes independent of $z$. It only occurs at specific spectral components $\omega$ (equal to the pulsation $\omega_{k}$ in Eq. 4) corresponding to solutions of the quasi-phase-matching relation 4. For these specific pulsation detunings $\omega_{k}$, each term of the sum in Eq. (22) leads to periodical amplification and deamplification phases along the DOF except for the uniform contribution corresponding to $q=m$. This last term therefore prevails over the other ones on the gain $g(\omega, z)$ for long enough propagation distances. Thus the linear gain of the $m^{\text {th }}$ spectral component can be approximated by this uniform term:

$$
g\left(\omega_{m}, z\right)=2 \gamma P_{p}\left|J_{m}\left(\frac{\beta_{M} \omega_{m}^{2}}{2 \pi / Z}\right)\right|
$$




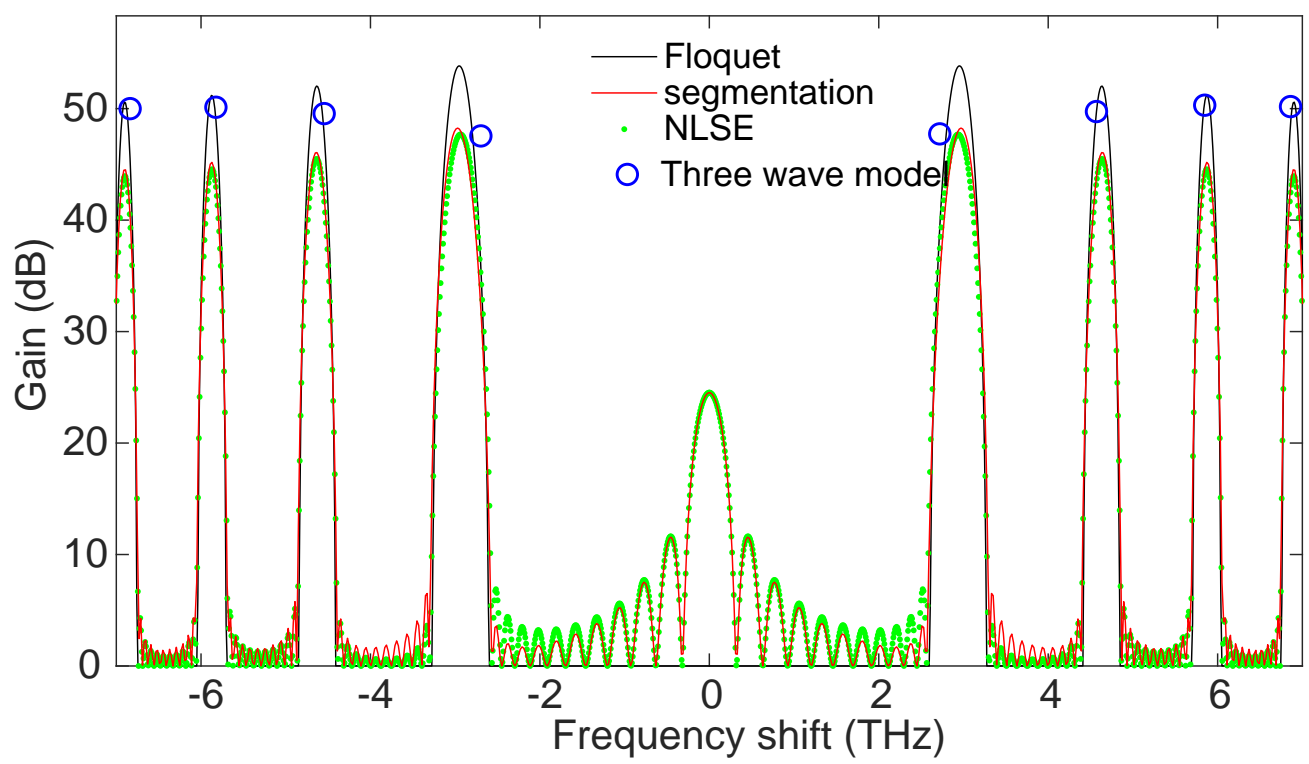

Fig. 9. Gain calculated form three-wave model Eq. (22) (blue circles), from Floquet theory (black curve), "segmentation" (red curve) and NLSE (green dots). Parameters correspond to the case studied in Ref. [45] $L=120 \mathrm{~m}$ $\bar{\beta}_{2}=1.2 \times 10^{-27} s^{2} / m, \beta_{M}=1.5 \times 10^{-27} s^{2} / m, \gamma=7 / W / K m . P=20 W$.

with $K_{m}=+\frac{\pi}{2}$ in order to maximize the uniform gain by adjusting $\theta\left(\omega_{m}, 0\right)$ [45]. The accuracy of this theoretical model has been evaluated by comparing its predictions with numerical simulations from the NLSE, the Floquet theory and the "segmentation model" (see caption of Fig. 1 for further information). A representative example of such comparison is reported in Fig. 9, with parameters taken from Ref. [45]. NLSE simulations has been performed by considering a pump and a small signal at frequency $\omega$ at input, and the gain is defined as the ratio between the output and the input signals. The parametric gain from Floquet theory has been calculated numerically, since with sinusoidal modulations Eq. (11) cannot be solved analytically. The gain has been calculated as $\exp [g(\omega) L]$, being $g(\omega)$ the parametric gain. This fact is responsible for the slightly higher gain calculated from Floquet, and the absence of ripples far away from phase matching, where the Floquet gain is strictly zero. In spite of this, a remarkably good agreement is observed between all the curves.

We now focus to the first spectral component $(m=1)$ ( higher oder mode evolutions can be found in Ref.[45]). Firstly, the longitudinal evolution of the gain is shown in Fig. 10 (a). Analytical results are represented in solid and dashed lines (Eq. (24)) and numerical ones in solid black line. The solid red line in Fig. 10 (a) corresponds to Eq. (22) including only $J_{-1}$ and $J_{0}$ and $J_{1}$ terms because all other Bessel functions have much lower contributions in this example. As can be seen, a pretty good agreement is achieved with numerical simulations by including these first three Bessel functions. The solid blue curve corresponds to the term of uniform gain (Bessel function $J_{1}$ only, $q=m$ ) which allows to give a very good estimation of the gain with a very simple expression (Eq. (24)). The evolution of the relative phase is shown in Fig. 10 (b) from theory (solid red curve, Eq. 23) and numerics (solid black curve). As can be see, a very good agreement is obtained. In each modulation period, the 


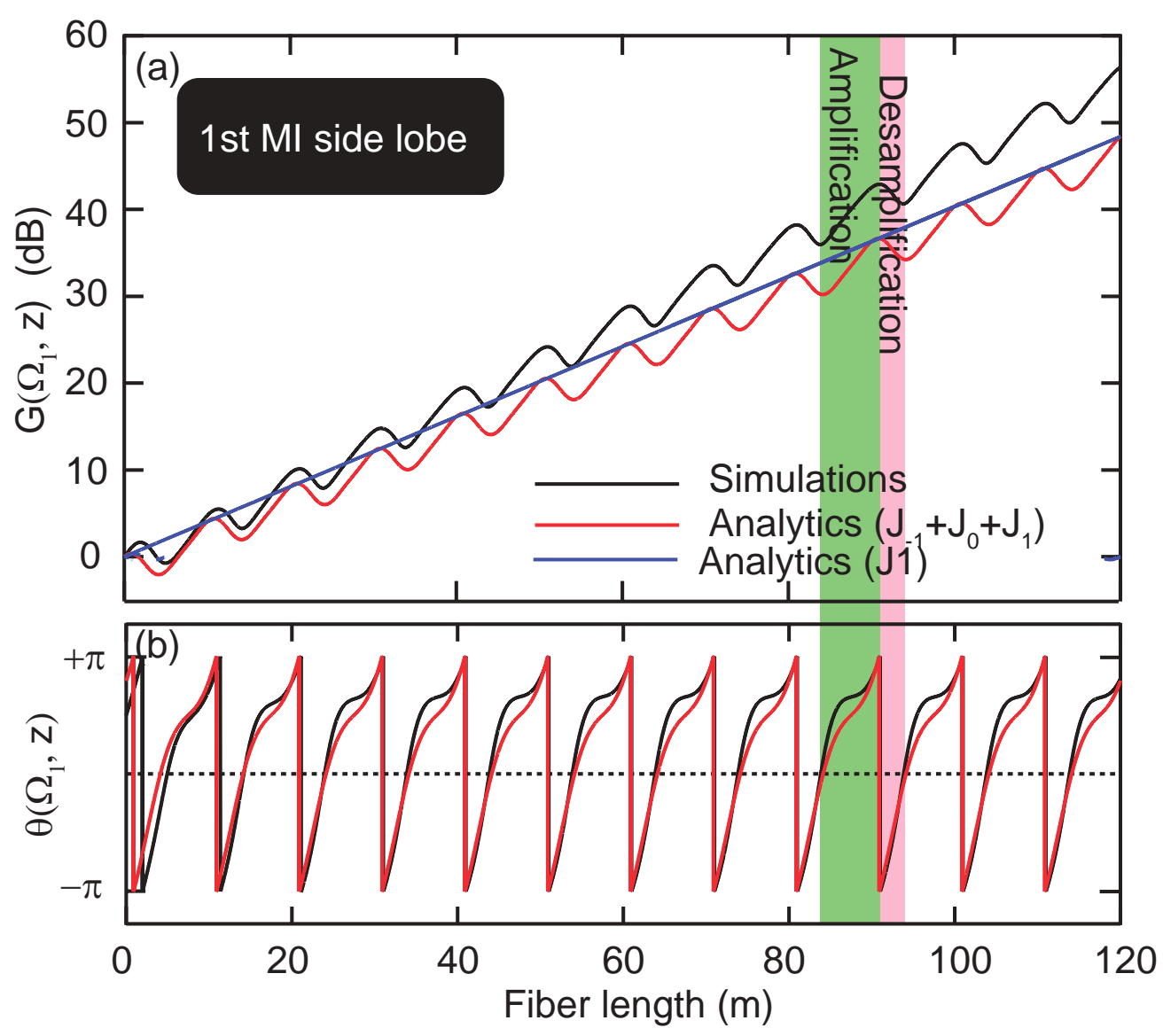

Fig. 10. Evolution of (a) the gain from Eqs. (22) and Eq. (24) and (b) the relative phase $\theta(z)$ from Eq. (23) of the first amplified frequency $(m=1)$ for a sinusoidally modulated optical fiber which parameters can be found in Ref. [45]. Analytical results are represented in red lines and numerical simulations in solid black line. The green area corresponds to amplification and the red one to deamplification over one period. Adapted from [45] [Physical Review A, 87, 013813 (2013)].

amplification phase is characterized by $0<\theta(\omega, z)<\pi$, the deamplification one has $-\pi<\theta(\omega, z)<0$ and the total phase shift being equal to $2 \pi$ per period. This model is also valid for higher order MI side lobes, as can be seen in Ref [45]. Finally, the very good agreement between our model and numerical simulations confirms the validity of our assumptions and the accuracy of our method. Moreover, the simple expression of the average gain (Eq. (24)) provides a rapid insight of the MI gain in DOFs. For instance in Ref. [45], we took benefit of this simple expression to adjust DOF parameter either to suppress or enhance a side band in a multi-band MI spectrum. Besides, it has been used in many other work to get a rapid insight of the MI dynamics $[50,54,55,58-60]$. 


\section{FIRST OBSERVATION OF MODULATION INSTABILITY IN DOFS}

Before reporting the first clear observation of MI in a periodically modulated fiber based system, we propose to review all fiber optic platforms that have/could be used to do so. They are gathered in Fig.11 through illustrative sketches and listed chronologically. The typical modulation period is indicated for each system in order to get a rough idea of MI frequency shifts that can be obtained in these systems. According to Eq. 4 , increasing/decreasing the modulation period leads to small/ large frequency shifts. The first system is a telecommunication network (Fig.11 (a)). It motivated the theoretical study of MI in fiber based periodic systems [37]. The periodicity is naturally provided by multiple stages of signal amplification. The period of modulation of a few tens of kilometers leads to MI sidebands within the GHz range, that thus degrades the transmission quality of telecommunication signals. Experimentally, one of the two first systems used to evidence MI in periodic fiber systems has been reported by Kikuchi et al.. [61]. They built a recirculating loop mimicking the propagation of light into a telecommunication network (see Fig.11 (b), the loop length is $40 \mathrm{~km}$ ). Whereas the most streaking feature of MI in periodic systems is the appearance of multiple quasi-phase matched sidebands, only one pair of sidebands was reported in this works. Thus, the evidence of MI due to periodicity was not crystal clear. The same year, Murdoch et al. [76] proposed another fiber based optical system by winding a piece of fiber around two holders separated by a few tens of centimeters, therefore modulating the fiber birefringence (see Fig.11 (c)). The frequency shift is a few Terahertz but again, only one pair of sidebands was been generated. The first unambiguous observation of MI in DOFs has been reported in a PCF in 2012 by Droques et al. [49] (see Fig.11 (d)) with a period of modulation of $10 \mathrm{~m}$. The fiber group velocity dispersion has been periodically modulated along the propagation axis by modulating the outer diameter by $\pm 7 \%$ of the average diameter value with a period of modulation of $10 \mathrm{~m}$. A quasi-continuous wave laser was launched in the normal dispersion region of this fiber, where no $\mathrm{MI}$ is expected to occur in uniform fibers. As a result of the periodic modulation of the dispersion, more than $10 \mathrm{MI}$ side lobes have been observed at the output of the fiber for a modest pump peak power of $20 \mathrm{~W}$ (Fig. 12). This experimental result has been confirmed by numerical simulations based on the NLSE including Raman effect (solid blue line in Fig. 12). In addition, the position of the QPM side lobes have been accurately predicted from the QPM relation (4, in vertical dashed lines in Fig. 12). These results constitute the first clear experimental observation of MI in a dispersion oscillating fiber. The versatility provided by the fabrication of these optical fibers allowed the experimental exploration of a large variety of regimes and opened the way to a wide field of investigations on MI process in these fibers since 2012. Main results following this first observation [49] are detailed in the next sections. Note that, following these works in single pass configurations, MI has also been investigated in passive fiber ring cavities made of two pieces of fiber (see Fig.11 (e)). Consequently, the pulse propagating inside the cavity experiences a periodic variation of the dispersion in addition with the periodic boundary conditions imposed by the cavity. It leads to a more complex dynamics than in single pass configurations with the appearance of instabilities of different nature (Turing and Faraday) that will be detailed in section 9. Finally, it has been recently reported that parametric instabilities can be observed in graded index multimode fibers. The periodicity originates from 

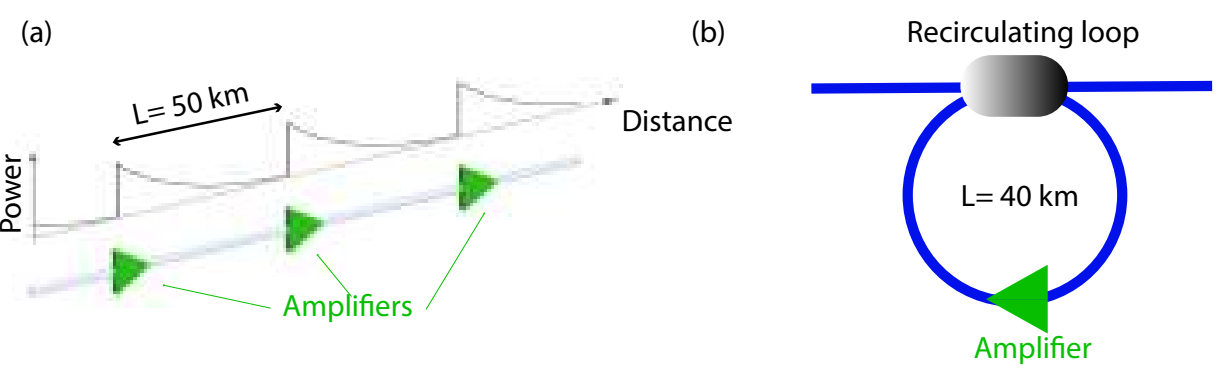

(c)

(d)
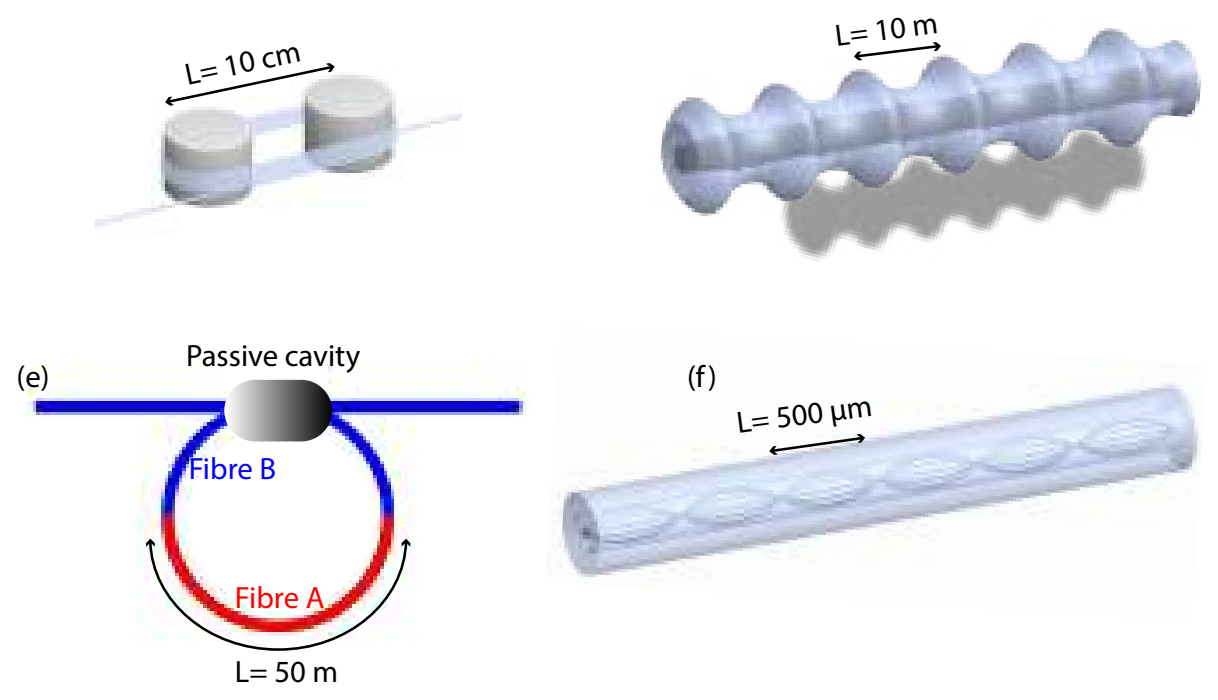

(f)

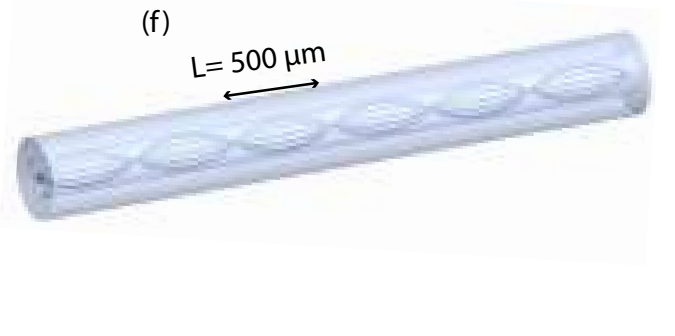

Fig. 11. Schemes representing different fiber optic systems in which one of the longitudinal parameters is modulated. (a) A telecommunication system with a periodic modulation of the power [37], (b) a recirculating loop aiming at mimicking a a telecommunication system [61], (c) a birefringent fiber with a periodic modulation of the effective index by applying a mechanical stress [76], (d) a PCF with a periodic modulation of the outer diameter during the drawing process [49], (e) a passive fiber ring cavity made of different fibers and (e) [77], and a graded index multimode fiber with a periodic variation of the field due to self imaging [78]. 


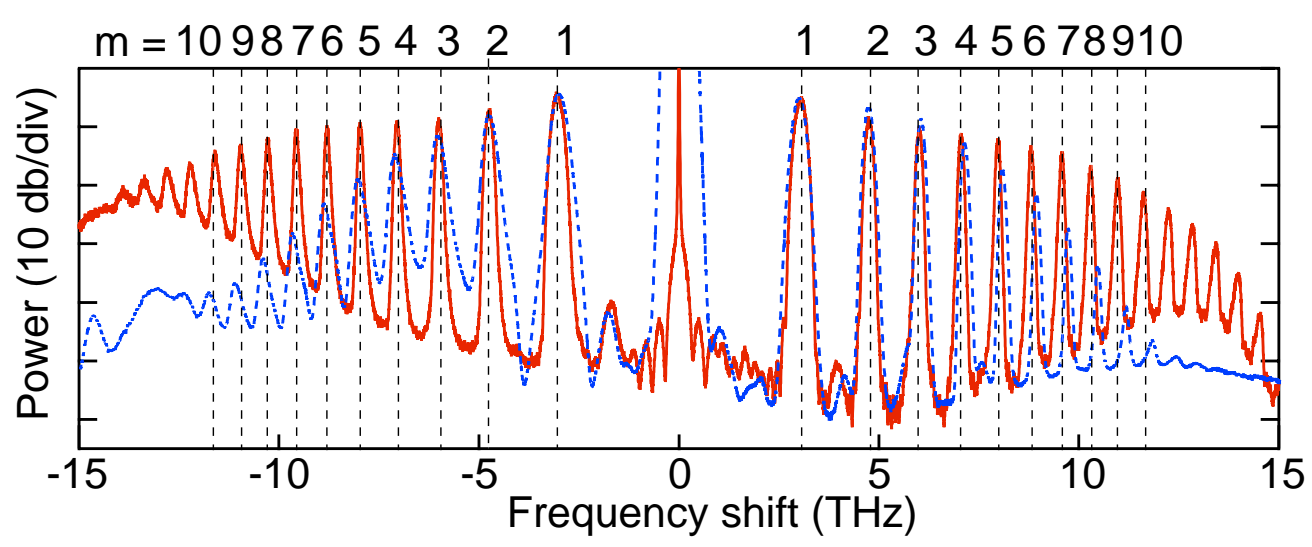

Fig. 12. Experimental (dashed blue lines) and numerical (solid red line) output spectra. Vertical dashed lines represent quasi-phase-matched frequencies obtained from Eq. (4) for $m=1$ to 10 .

the self-imaging process of the field propagating in these fibers. The period of modulation is a few hundreds of micrometers leading to large frequency shifts of tens of terahertz (see Fig.11 (f)) [78].

\section{MODULATION INSTABILITY IN DOFS IN THE UNDEPLETED PUMP REGIME}

In this section, we summarize the overall results obtained in the limit of the undepleted pump approximation.

\section{A. Correlation in energy between MI side lobes}

The most striking feature of MI in DOFs is that many side bands can be destabilized. However, coming back to the simple quantum mechanical picture of MI process where two pump photons are annihilated into one signal and one idler photon, one may ask if this simple interpretation is still relevant in these fibers. In other words, each side band pair is associated to a quasi-phase matching parametric process, but are these parametric processes independent of each other? In order to reply to this question, we carried out numerical simulations by integrating the stochastic generalized nonlinear Schrödinger equation [7]. We run more than 100 simulations with random initial conditions (half photon per temporal mode) and we calculated the total energy located in each MI side lobe, to finally estimate their relative correlations. We investigated a DOF with a sinusoidal modulation having a period of modulation of $10 \mathrm{~m}$ and pumped by a continuous wave. All other parameters are listed in Fig. 13 's caption. They have been adjusted in order to generate only to main side lobes $(m=1$ and $m=2$ ) to obtain the simplest configuration to carry out this study. The average output spectrum is shown in Fig. 13 (a). It exhibits a first strong QPM MI order at 3.6 THz labeled $Q_{1}$, and a second one labeled $Q_{2}, 10 \mathrm{~dB}$ lower, at 5.7 $\mathrm{THz}$. An harmonic, resulting from the beating of $Q_{1}$ with the pump is also generated at 7.2 THz and labeled $H_{1}$. The respective conjugated waves appear symmetrically from the pump and are represented with stars, $Q_{1,2}^{*}$ and $H_{1}^{*}$ respectively. Fig. 13 (b), (c) and (d) represents scattergrams between two conjugate side lobes $Q_{1}-Q_{1}^{*}$ and $Q_{2}-Q_{2}^{*}$ and between distinct ones $Q_{1}-Q_{2}$. The degree of correlation is almost equal to one (Fig. 13 (b) and (c)) when 

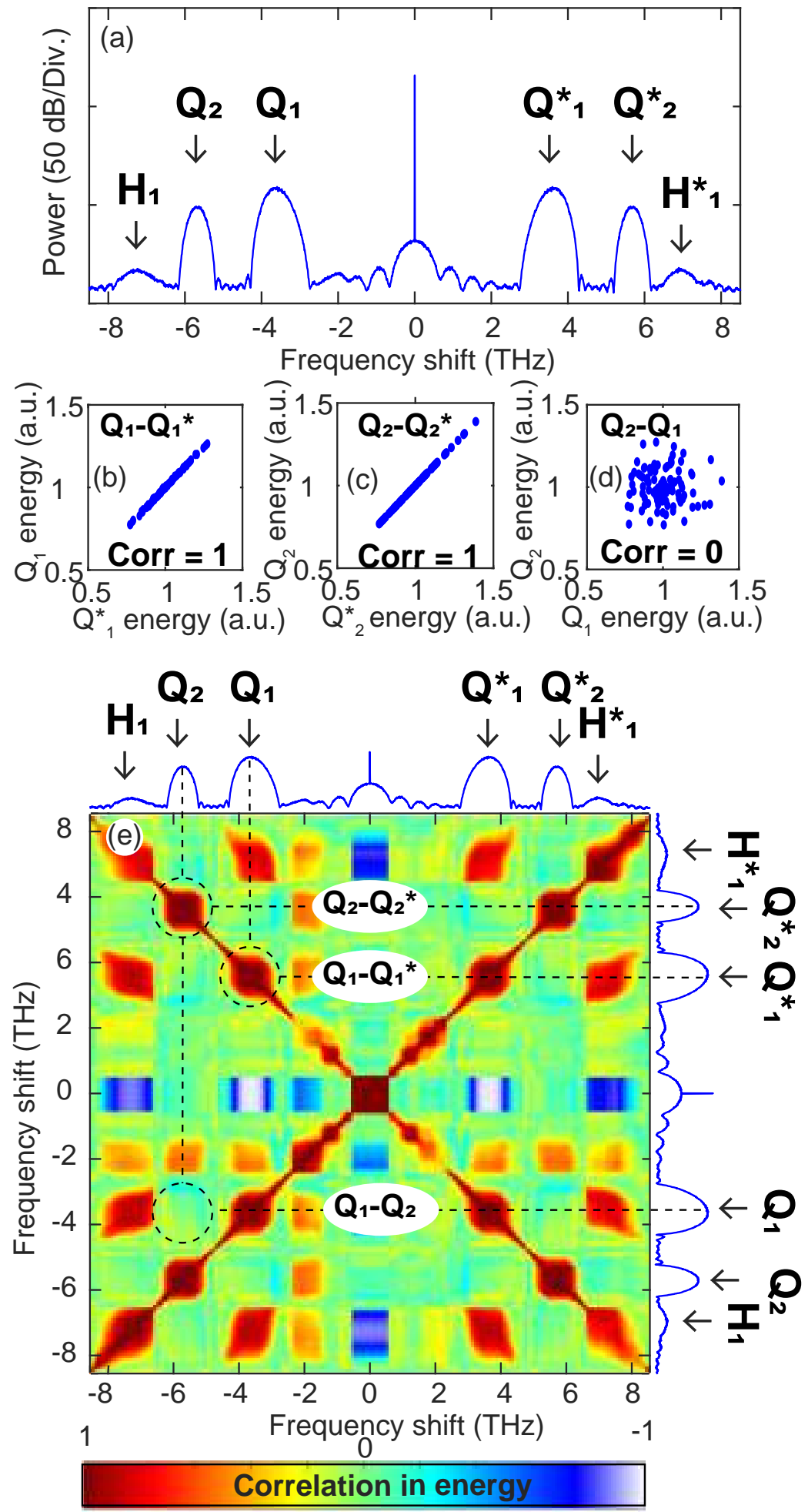

Fig. 13. (a) Average spectrum. (b), (c) and (d) Scattergram between $Q_{1}-Q_{1}^{*}$, $Q_{2}-Q_{2}^{*}$ and $Q_{1}-Q_{2}$ respectively. (e) Energy correlation map. $\bar{\beta}_{2}=0.7 \times$ $10^{-27} \mathrm{~s}^{2} / \mathrm{m}, \beta_{2}^{A}=+1.8 \times 10^{-27} \mathrm{~s}^{2} / \mathrm{m}, \beta_{3}=0.52 \times 10^{-40} \mathrm{~s}^{3} / \mathrm{m}, \beta_{4}=-1.1 \times$ $10^{-55} \mathrm{~s}^{4} / \mathrm{m}, \gamma=5.5 / \mathrm{W} / \mathrm{km}, P_{P}=39 \mathrm{~W}, \alpha=1 \mathrm{~dB} / \mathrm{km}, \mathrm{Z}=10 \mathrm{~m}$, and $L=68 \mathrm{~m}$. 
considering conjugate side lobes while it is zero for a cross combination of MI side lobes (Fig. $13(\mathrm{~d})$ ). In order to get an overview of the degree of correlation involving other MI side lobes or harmonics couples, we calculated the energy correlation map. It is defined as follows:

$$
\rho=\frac{<E\left(f_{1}\right) E\left(f_{2}\right)>-<E\left(f_{1}\right)><E\left(f_{2}\right)>}{\sqrt{\left(<E\left(f_{1}\right)^{2}>-<E\left(f_{1}\right)>^{2}\right)\left(<E\left(f_{2}\right)^{2}>-<E\left(f_{2}\right)>^{2}\right)}}
$$

where $E(f)$ is the array of energy at a particular frequency $f$, calculated in a $500 \mathrm{GHz}$ frequency span. Brackets angle represent the average over the ensemble. The spectral correlation varies over the range $-1<\rho<1$ (see colorbar in Fig. 13 (e)). This correlation map representation has been widely used in correlated photon pair generation for quantum information [79], and more recently to investigate noise in SCs $[80,81]$ or intermodal correlation in MI spectra [82]. As can be seen, this representation gives similar results for side lobe couples which scattergram are shown in Fig. 13 (b), (c) and (d). They are highlighted in the energy correlation map with dashed lines in Fig. 13 (e). This 2D plot is extremely useful because we can directly see the degree of correlation between any side lobe orders. For instance, we see that the harmonic $H_{1}$ is strongly correlated with $Q_{1}, Q_{1}^{*}$, and the pump waves involved the four wave mixing from witch it originates. More generally, all complex conjugate couples are strongly correlated while cross combinations are weakly. These investigations have been validated by experiments [54]. In order to record fluctuations in energy in each QPM side lobe from shot to shot, it is necessary to record spontaneous spectra. The use of an optical spectrum analyser is useless because its integration time is well longer than the period of the pump train. It has been done by means of the dispersive Fourier transform technique [83], a very useful technique to record instantaneous spectra in the context of MI [82], rogue waves [15] or supercontinuum [80, 81] in uniform fibers. With this setup we have been able to calculate the degree of correlation between conjugate side band pairs and cross combination pairs. These experimental results have been confirmed by numerical simulations (not shown here, see Ref. [54]). Basically, we get the same conclusion that those derived from numerical simulations shown in Fig. 13, that is to say, conjugate side lobes are strongly correlated while other cross combinations are not. Finally, we can conclude that MI side band pairs originate from parametric processes that can be considered independent from each others.

\section{B. Weak dispersion region: impact of the fourth order dispersion term}

Most of theoretical and experimental investigations of MI in DOFs have been achieved in relatively large dispersive fibers where the development up to the second order of the propagation constant provides a satisfactory description of the process. However, it is well known in uniform fibers that by working in low dispersion region requires to consider higher order dispersion terms to accurately describe the dynamics of the process [84]. More specifically, it is demonstrated theoretically in Refs $[85,86]$ and experimentally in Refs. [87, 88] that a development up to the fourth order dispersion term is needed to explain why MI is observable in normal dispersion region of uniform optical fibers. In DOFs, similar considerations can be done. When the average group velocity dispersion is very weak, i.e. when the pump wavelength is located in the 

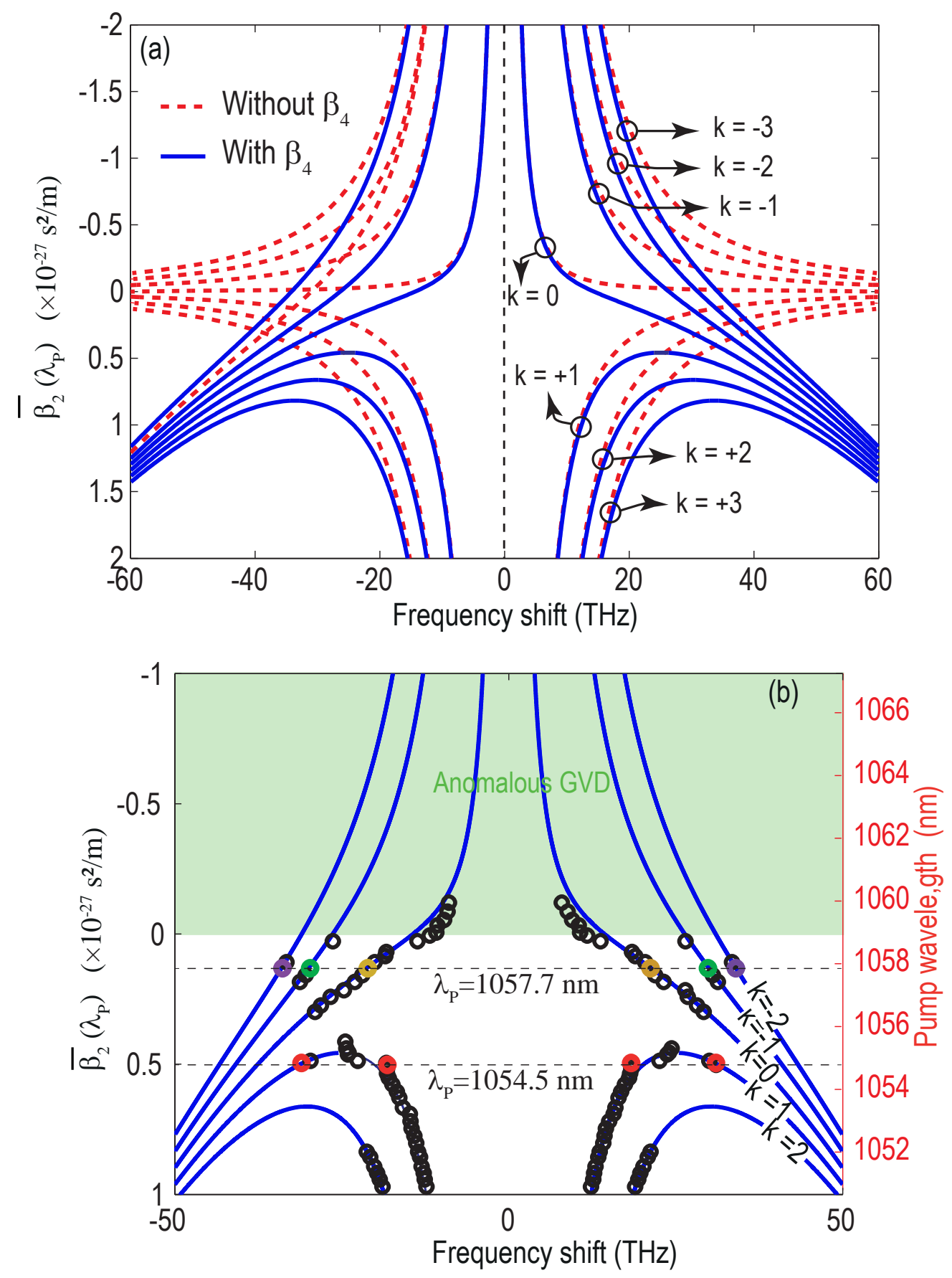

Fig. 14. (a) Quasi-phase matching curves calculated from Eq. (26) with and without the $\beta_{4}$ term (blue solid and red dashed lines, respectively) as a function of average GVD . (b) QPM curves calculated from Eq. (26) (solid line) and measurement of MI sideband frequencies done by tuning the pump wavelength (markers). Adapted from [50] [Optics Letters, 38, 3464 (2013)]. 
vicinity of the average zero dispersion wavelength of the fiber, higher order dispersion terms must be accounted for. In Ref. [50], we showed that QPM relation in the vicinity of the average zero dispersion value reads :

$$
\bar{\beta}_{2} \omega^{2}+\frac{\bar{\beta}_{4}}{12} \omega^{4}+2 \gamma P=2 m \pi / Z,
$$

with $\bar{\beta}_{4}$ the average value of the fourth order dispersion. Consequently, this additional term leads to the generation of a new family of MI frequencies arising from a combination of fourth order dispersion and longitudinal periodic dispersion. It is illustrated in Fig. 14 (a) where the evolution of solutions of Eq. (26) (limited to $m=0, \pm 1$ for the sake of clarity) as a function of the average value of the dispersion are represented with (solid blue lines) and without (dashed red lines) $\beta_{4}$. The most important feature arises in the normal dispersion region where, for each value of average GVD, the branch corresponding to $m=+1$ gives only one solution when $\beta_{4}$ is neglected (dashed red line), whereas a second one exists with $\beta_{4}$ (solid blue line). In addition, similarly to what occur in uniform fibers, a solution corresponding to $m=0$ exists in this dispersion region. To generalize, two solutions corresponding to $m>0$ appear in the normal dispersion region in addition with the single solution corresponding to $m \leq 0$. These predictions have been confirmed experimentally with an excellent agreement with numerical simulations and theoretical predictions (see Fig. 14 (b)) [45]. These investigations were limited to relatively weak amplitude of modulation, but a theoretical study of Armaroli and Biancalana [48] revealed that for large amplitude of modulations the conventional MI is suppressed and for large negative values of $\beta_{4}$ the side bands can merge together.

\section{Other modulation formats}

From a practical point of view DOFs are not limited to sinusoidal modulation formats. Limitations are imposed by the drawing tower itself. They are due to (i) the inertia of the fiber tower that fixes the shortest length of the modulation period and (ii) the deformation of the fiber structure that gives the maximum amplitude of variation. Note that it is relatively tricky to put numbers on these limit values since they depend many drawing parameters and these limits depend on each other. In order to illustrate the capability of our drawing tower, we fabricated DOFs with two different modulation formats. It also gave us the opportunity to develop specific theoretical tools to investigate different formats from the basic sinusoidal one. Firstly, a DOF with a sine modulation whose amplitude is modulated by another sine function has been fabricated [53]. The evolution of MI gain spectra versus the ratio between the oscillation period $Z_{1}$ (short oscillation) and the modulation period $Z_{2}$ (long oscillation) of the dispersion is represented in Fig. 15 (a). It has been calculated from the "segmentation method" described in Fig. 1 's caption. We see that decreasing the modulation period leads to a splitting of original side lobes into multiple ones of lower gain. These numerical simulations where confirmed by a theoretical study based on the truncated three wave model by following a procedure similar to the one developed in Ref. [45]. Finally, we get an analytical expression allowing to predict the position of these new side bands (see Ref. [53] for more details). In order to validate these theoretical investigations we fabricated two different DOFs: a standard DOF with a sinusoidal modulation 
(a)

Gain (2 dB/div.)
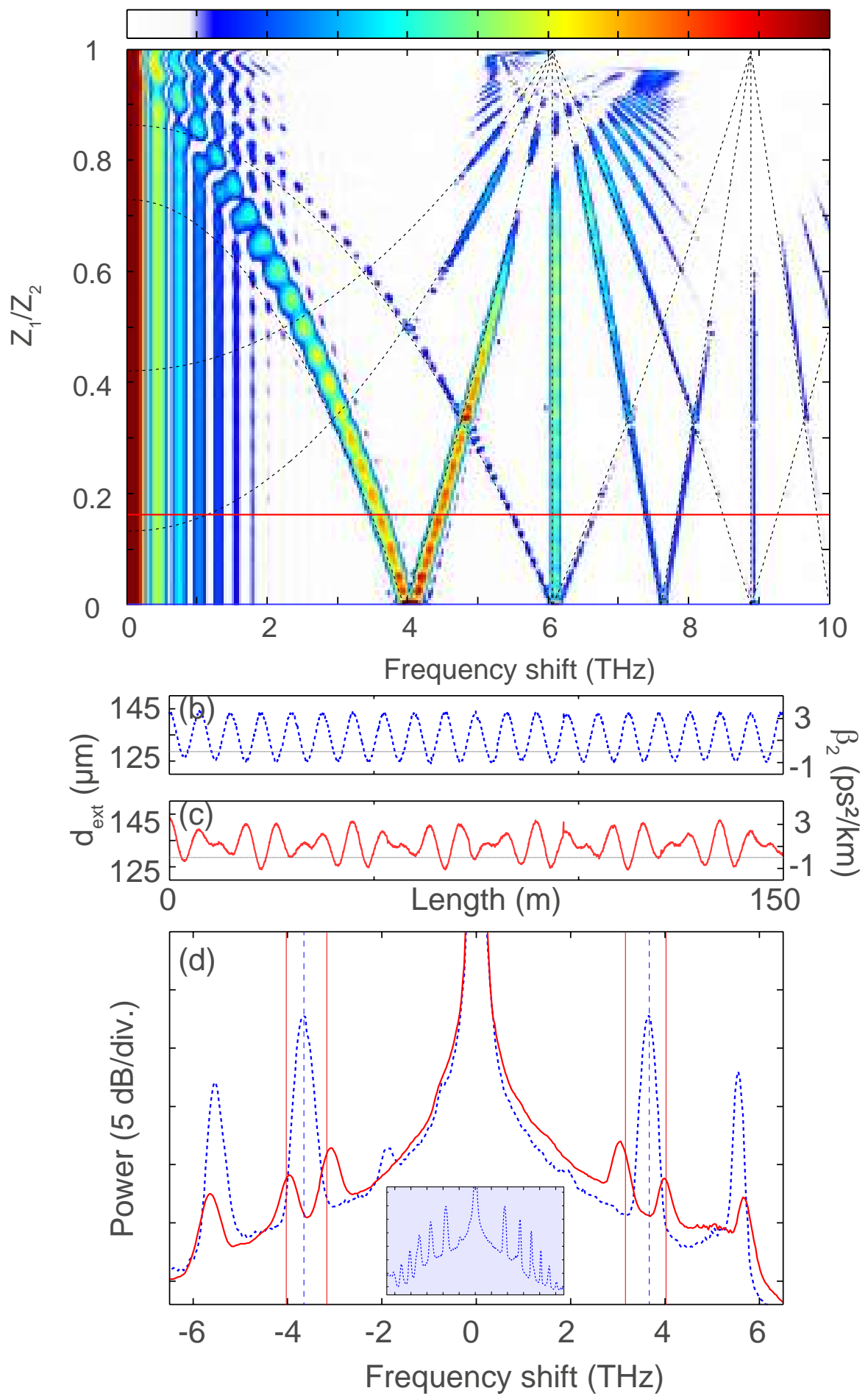

Fig. 15. (a) Gain spectrum in amplitude modulated fibers as a function of the $Z_{1} / Z_{2}$ ratio $\left(Z_{1}\right.$ is fixed and equal to $7.5 \mathrm{~m}, Z_{2}$ varies from $\infty$ to $Z_{1}$ ). The dotted lines represent the predicted positions of the sidelobes maximum. External diameter and dispersion profile of (b) the reference oscillating fiber, and (c) the amplitude modulated fiber. (d) Experimental spectra out of the reference fiber (blue dotted line) and the amplitude modulated fiber (solid red line). The red arrows point out the predicted positions of the first two sidelobes in the amplitude modulated fiber. Adapted from [53] [Optics Express, 23, 3869 (2015)]. 
of its diameter for reference, and a DOFs that is modulated in amplitude. The spectrum recorded at the output of the reference fiber $\left(Z_{1} / Z_{2}=0, F\right.$ ig. 15 (b)) is represented in dashed blue lines in Fig. 15 (d). As in previous works $[45,49]$, the positions of the side bands is very well predicted by Eq. 4 (vertical dashed blue lines in Fig. 15 (d)). In the amplitude modulated fiber (period of modulation of $45 \mathrm{~m}$ so $Z_{1} / Z_{2}=0.16$, see Fig. 15 (c)), the first side band splits into two side bands (red curve in Fig. 15 (d)) and their positions are very well predicted by the theory developed in Ref. [53] (vertical solid red lines in Fig. $15(d))$.

In Ref. [51], a radically different periodic modulation of the GVD has been proposed. We choose to fabricate a DOF characterized by a dispersion that has the form of a periodic train of Dirac delta spikes. Figure 16 (a) represents the evolution of the outer diameter of this delta Dirac fiber as a function of the fiber length. Due to technological limitations of the drawing process, a series of Gaussian pulses have been chosen to approximate the ideal Dirac delta comb. The maximum value of modulation of the diameter is $175 \%$ of the average diameter value corresponding to a variation of $3500 \%$ of the dispersion by pumping in the vicinity $(1052.5 \mathrm{~nm})$ of the average zero dispersion wavelength of the fiber $(\simeq 1053 \mathrm{~nm}$ ). The full width at half maximum of the gaussian pulse is $60 \mathrm{~cm}$. We pushed the drawing tower at the limit to fabricate these fibers, that thus illustrates the limitations of the capabilities of these systems for this specific example $(d / \Lambda=0.48$ and $\Lambda=3.45 \mu \mathrm{m})$. A quasi continuous wave laser was launched in the average normal dispersion of this fiber, and more than 7 quasi-phase matched side lobes due to the periodic variation of the dispersion have been observed. Additionally, an analytical expression of the parametric gain has been derived from perturbative theory [51]. We found a very good agreement between these predictions (green dashed lines in Fig. 16 (c)), experimental results (Fig. 16 (b)) and numerical simulations (Fig. 16 (c)).

These results gives a snap shot of the potential of our drawing tower to fabricate a large variety of DOFs. More generally, this illustrates that optical fibers could constitute an interesting platform for achieving experimental investigations of fundamental physical phenomena in delta Dirac fibers, which is a fundamental and widespread modulation format, encountered in a large variety of physical systems [89-91]. It may initiate or motivate the use of such waveguides to perform experimental investigations in these contexts.

\section{PARAMETRIC AMPLIFICATION IN DOFS}

MI leads to the amplification of a small perturbation provided that a phase matching or quasi-phase matching relation is fulfilled. In all previous results, MI has been seeded by random initial conditions. However, if the process is seeded by a weak coherent signal, it will be amplified through MI process and in that case we usually refer to as fiber optical parametric amplification [20,21].

These amplifiers have been widely investigated in the context of telecommunication applications because of their large bandwidths and large gain bands [20]. The drawing process of optical fibers suffers from different sources of perturbations or imperfections leading to spurious longitudinal variations of the fiber dispersion. The impact of these variations have been investigated in the context of fiber optical parametric amplifiers in uniform fibers [92-94]. It is shown that they lead to a lowering of the parametric gain that is detrimental for 

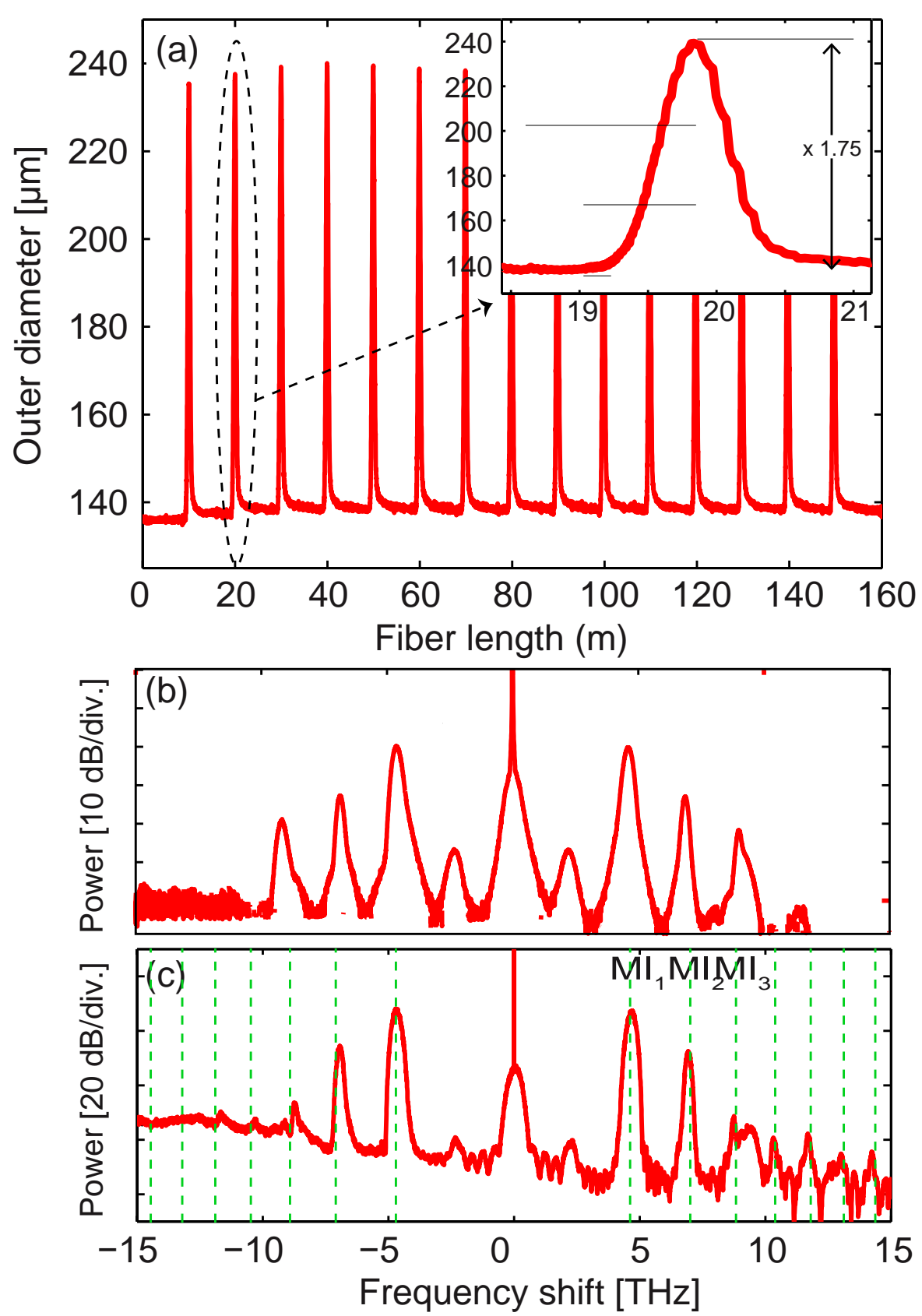

Fig. 16. (a) Outer diameter evolution versus fiber length. Inset: Snapshot on one peak. (b) Spectra recorded at the fiber output and (c) numerical simulations. Adapted from [51] [Physical Review A, 92, 013810 (2015)]. 


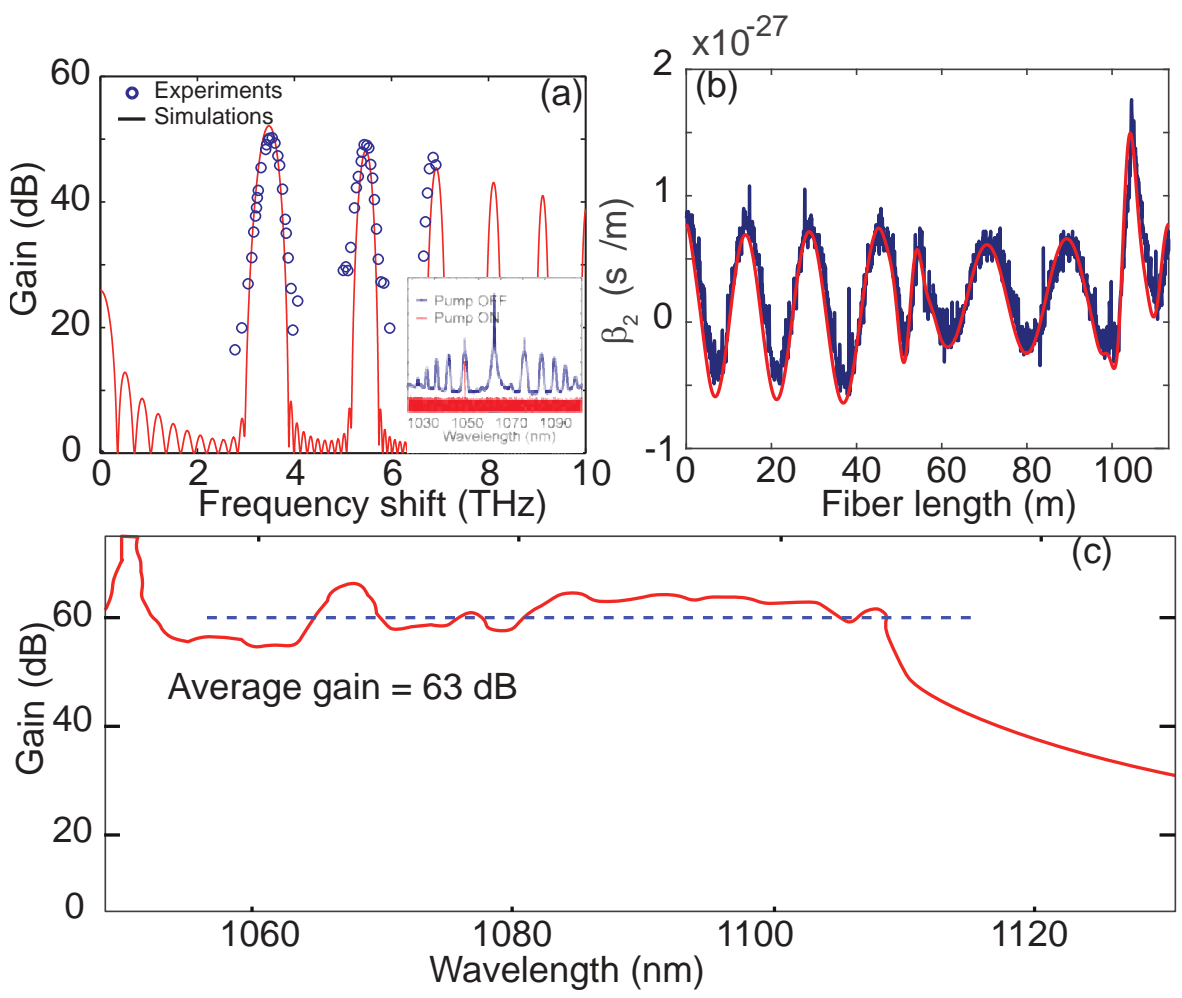

Fig. 17. (a) Gain curves, experimental results (circles), numerical simulation (solid line). Parameters: pump power $26 \mathrm{~W}$, signal power $10 \mu \mathrm{W}$, fiber length $120 \mathrm{~m}$, period of modulation $10 \mathrm{~m}$, amplitude of modulation $2 \times 10^{-27} \mathrm{~s}^{2} / \mathrm{m}$ and linear loss $8 \mathrm{~dB} / \mathrm{km}$. (b) Optimal longitudinal evolution of the group velocity dispersion to obtain the flat gain curve shown in (c). (c) Gain curve at the output of fiber with dispersion shown in (b) for $30 \mathrm{~W}$ pump power.

applications. Attempts have been made to design optical fibers less sensitive to these fluctuations that allow a significant improvement of the efficiency of amplification in standard highly nonlinear fibers [95] for telecommunication amplifications or in PCFs at $1 \mu \mathrm{m}$ for short pulse amplification [96]. Here, we propose to show that is possible to take benefit from controlled periodic dispersion variations in DOFs to get wide band fiber optic parametric amplifiers. Seeding a DOF with a small coherent signal can lead to its amplification with a degradation of its noise figure of at least $3 \mathrm{~dB}$ but without additional distortions compared to uniform fibers as they relied both on parametric p rocesses. We performed these experiments in a DOF described by the parameters listed in Fig. 17 caption. The experimental setup is similar to the one used for MI experiments, except that we combined a small signal with the pump at the input of the fiber. The on/off gain was measured at the output of the fiber by using an optical spectrum analyzer (see pump on/off spectra in Fig. 17(a)). By tuning the signal wavelength, we measured the gain curve (Fig. 17(a) in circles). We see that large gain values around $50 \mathrm{~dB}$ have been measured on the top of the first QPM MI side lobe of this DOF. An excellent agreement is obtained with numerical simulations (solid red line). These basic experiments illustrate that these systems can indeed be used as amplifiers, similarly to uniform fibers. Form a practical point of view, the use of DOFs as parametric 
amplifiers requires large and continuous gain bands while it is composed of multiple distinct QPM side bands. To overcome these limitations, we designed a special DOF whose period and amplitude of modulation have been optimized with a genetic algorithm [97]. The longitudinal evolution of this optimized DOF is represented in Fig. 17(b). By using a pump of $30 \mathrm{~W}$ peak power, we have been able to obtain a nearly flat (7 dB of ripple), wide gain band (12 $\mathrm{THz}$ ) amplifier with more than $60 \mathrm{~dB}$ of gain [97] (Fig. 17(c)). This DOF based amplifier should allow the amplification of very short pulses $<50 f s$ duration without being restricted by the gain narrowing process encountered in doped Ytterbium amplifiers [98]. These investigations have been carried out in PCFs at $1 \mu \mathrm{m}$ but similar results could be obtained at $1.5 \mu \mathrm{m}$ in standard optical fibers for telecommunication applications as shown in the context of frequency conversion in Ref. [99].

\section{NONLINEAR STAGE OF MODULATION INSTABILITY IN DOFS}

The previous sections were devoted to the undepleted or linear regime of MI in DOFs. In other words, we assumed that the power of the side bands can always be considered as negligible with respect to the pump power. We will see in this section that by increasing the pump power and/or by using longer fiber lengths, the power of these side bands is no longer negligible and the dynamic of the system is strongly modified. Consequently, we will see below that nonlinear regimes such as spurious four wave mixing processes between the QPM side bands, Fermi Pasta Ulam recurrence or solitonic train generation can also be observed in DOFs.

\section{A. Parasitic FWM interactions}

The evolution of the output spectrum as a function of the pump power has been investigated in Ref. [60]. They showed that by increasing the pump power, a complex nonlinear competition takes place between QPM side lobes, harmonics and additional parasitic four wave mixing waves.

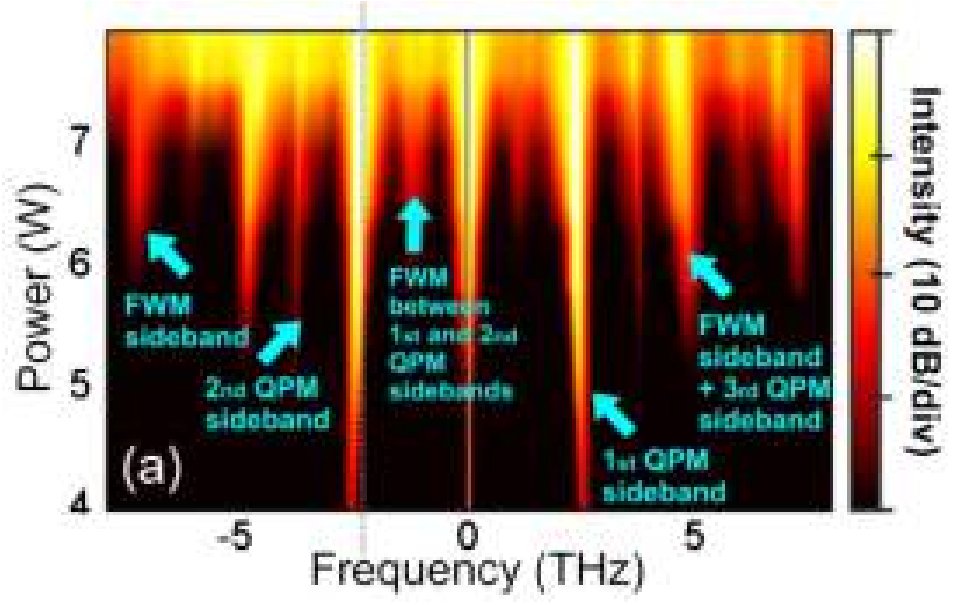

Fig. 18. Contour plot of experimental spectra versus input pump power. The fiber was sinusoidally modulated with a period of $20 \mathrm{~m}$ and the average dispersion value is $0.74 \mathrm{ps}^{2} / \mathrm{km}$. Adapted from [60] [Optics Letters, 38, 5361 (2013)]. 
A more precise picture is given in Fig. 18 that is extracted from these works. It represents the evolution of the output spectrum as a function of the pump power from experimental measurements. For relatively weak pump power level, below $5 \mathrm{~W}$, only the first order side band is destabilized at $f_{Q P M_{1}}=$ $\omega_{Q P M_{1}} /(2 \pi)=2.4 \mathrm{THz}$. By progressively increasing the pump power, around $6 \mathrm{~W}$, an harmonic appears at about $f_{H_{1}}=4.8 \mathrm{THz}$ as well as the second order QPM mode at about $f_{\mathrm{QPM}_{2}}=3.8 \mathrm{THz}$. Further increasing the pump power up to $6.5 \mathrm{~W}$ leads to a more complex dynamics because the third QPM mode starts growing at $f_{\mathrm{QPM}_{3}}=5 \mathrm{THz}$ and overlaps with the first harmonic. In addition, a spurious four wave mixing process involving the first and the second QPM side bands generate two new side bands at $f_{F W M_{1}}=1.2 \mathrm{THz}$ and $f_{F W M_{2}}=5 \mathrm{THz}$ fulfilling this energy conservation relation: $\omega_{Q P M_{1}}+\omega_{Q P M_{2}}=\omega_{F W M_{1}}+\omega_{F W M_{2}}$. As a result, a complex mix between QPM bands, harmonics and spurious four wave mixing process takes place, which finally evolves toward a continuum of frequency for higher pump powers.

\section{B. Fermi Pasta Ulam recurrence and out of band gain in average normal dispersion DOFs}

As described in section 4, the theoretical treatment of the early stage of MI in DOFs can be accurately achieved via Floquet analysis [38, 39, 42] or truncated three wave analysis [45]. The validity of these approaches have been confirmed by lot of numerical and experimental results, however, they are essentially limited to determine the region of parametric instability and can not accurately describe the dynamics of subsequent nonlinear MI stages past the linearized growth of the unstable modes. In that case, a more sophisticated approach based on a combining mode truncation and averaging methods is required to account for the whole complex dynamics of this nonlinear system. This procedure has been developed in Ref. [52]. Quite remarkably, such an approach reveals the existence of breather solutions separating different FPU recurrent regimes. Our theory also shows that optimal parametric amplification unexpectedly occurs outside the bandwidth of the resonance (Arnold tongues) arising from the linearized Floquet analysis.

In uniform fibers, FPU recurrence of MI [8] is commonly associated with the underlying integrable structure of the focusing NLSE that rules the propagation in the anomalous GVD regime [73, 75, 100]. However, similar recurrent behavior and a full homoclinic structure are also possessed by non-integrable models such as the coupled NLSEs that model polarization MI in birefringent fibers taking place in the normal GVD regime [101, 102]. Nevertheless, the results of Ref. [52] are the first that point out the remarkable and counter-intuitive fact that the recurrence behavior can be observed also in the scalar case in a nonfocusing regime (normal GVD), due to an underlying geometric structure of parametrically resonant MI in the depleted regime. The resulting recurrence is briefly illustrated in Fig. 19(a) which represents the longitudinal evolution of the pump and signal powers in a DOF with normal average dispersion. Two cycles of recurrence can be observed with a period of about 25 in normalized units in addition with very short periodic variations of the order of 1 superimposed on these long scale variations on both traces. They originate from the QPM process and this short period is equal to the period of GVD modulation of the fiber.

The second a priori unexpected prediction of our theory is that the maximum 

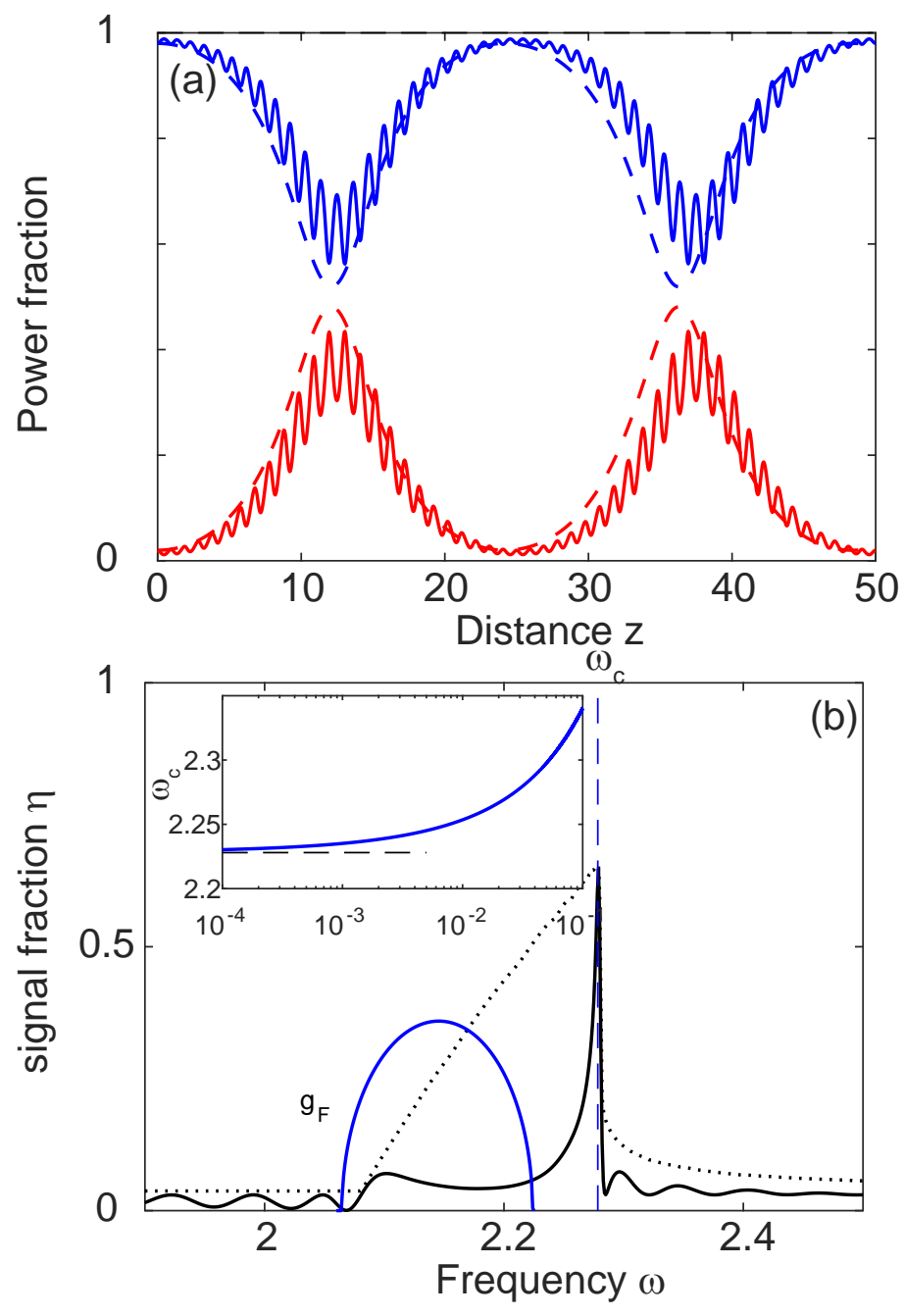

Fig. 19. (a) Evolution of extracted pump (blue line) and sideband power (red line) fractions from numerical simulations of the NLSE (1) compared with those from the average model (dashed lines), (Eq. (10) of Ref. [52]). (b) Output sideband fraction $\eta$ vs. frequency detuning $\omega$ from NLSE numerical integration at fixed $z$ (black solid line; red solid line is the maximum achievable conversion along $z$ ) with superimposed small-signal gain (blue). Inset: optimal frequency $\omega_{c}$ vs. input signal fraction $\eta_{0}$. Adapted from [52] [Physical Review Letter, 117, 013901 (2016)]. 
amplification experienced by an input signal with finite power is located outside the small signal gain bandwidth predicted by the Floquet analysis. This is illustrated in Fig. 19 (b) where the black line corresponds to the nonlinear regime and the blue line to the linear one (Floquet theory). We can see that the maximum growth experienced by the signal is located at $\omega=\omega_{c}=2.28$ (normalized units) while the Floquet theory predicts a stable pump (i.e., no gain) for $\omega \geq 2.224$. Here such a behavior is mediated by the existence of unstable eigen modulations of the process [52]. The frequency of optimal parametric conversion $\omega_{c}$ is always located outside the Floquet band and tends to its high frequency edge as the input power fraction $\eta_{0}$ get progressively smaller, see inset in Fig. 19 (b).

\section{Soliton formation and multiple dispersive wave radiation}

In DOFs, the additional degree of freedom provided by the periodic modulation of the dispersion allows for the observation of MI while pumping in the average normal dispersion region of fibers. In order to illustrate this stricking property, most of investigations have been realized in this average dispersion regime (see most of references related to DOFs cited in this review). However, pumping in the anomalous dispersion region also reveals clear signature of the periodic variation of the dispersion through the growth of higher order MI side bands $(m \neq 0)$ in addition to conventional ones $(m=0)$, that can be observed in uniform fibers. This was illustrated from numerical simulations in Fig. 2 (b) at the beginning of this review, but it has never been observed experimentally. In Ref. [57], we report the investigation of MI in DOFs in this average anomalous dispersion regime in the linear and nonlinear regimes of MI. In Fig. 20 (a), we represented the spectrum at the output of the DOF by pumping in the average anomalous dispersion region in the linear regime of MI (undepleted approximation). Two strong phase matched MI side lobes in addition with weak QPM side lobes are observed at the output of this fiber. The amplitude of conventional side bands is well above the amplitude of QPM ones because the parametric gain they experience is larger. Indeed, parametric gain in uniform fibers for perfect phase matched waves is equal to $g=2 \gamma P_{p}$. It is always larger than $g\left(\omega_{m}, z\right)=2 \gamma P_{p}\left|J_{m}\left(\frac{\beta_{M} \omega_{m}^{2}}{2 \pi / Z}\right)\right|$, the parametric gain for quasi phase matched waves in DOFs [45].

By further increasing the pump power up to $8 \mathrm{~W}$, a more complex and unexpected spectrum is recorded since it is composed of many side lobes that are asymmetric from the pump (red curve in Fig. 20 (a)). In order to explain their origin, we have shown in Ref. [57], that by increasing the pump power a train of soliton pulses is generated. Consequently, each soliton is destabilized by the periodic variation of the dispersion and sheds energy to multiple resonant radiations the positions of which are well predicted by a quasi-phase matching relation. The latter has been derived in the context of the propagation of a single soliton in DOFs [103] from a perturbative theory and writes as:

$$
-\Delta k_{1} \omega+\overline{\beta_{2}} \omega^{2} / 2+\overline{\beta_{3}} \omega^{3} / 6+\overline{\beta_{4}} \omega^{4} / 24-\gamma P_{S} / 2=2 m \pi / Z
$$

where $\bar{\beta}_{2,3,4}$ are the average second, third and fourth dispersion orders, $\Delta k_{1}$ arises for from the deviation of the actual group-velocity from the natural one [104], and $P_{S}$, is the soliton peak power. It is important to note that this quasi-phase matching relation (Eq. 27) has nothing to do with QPM relation 

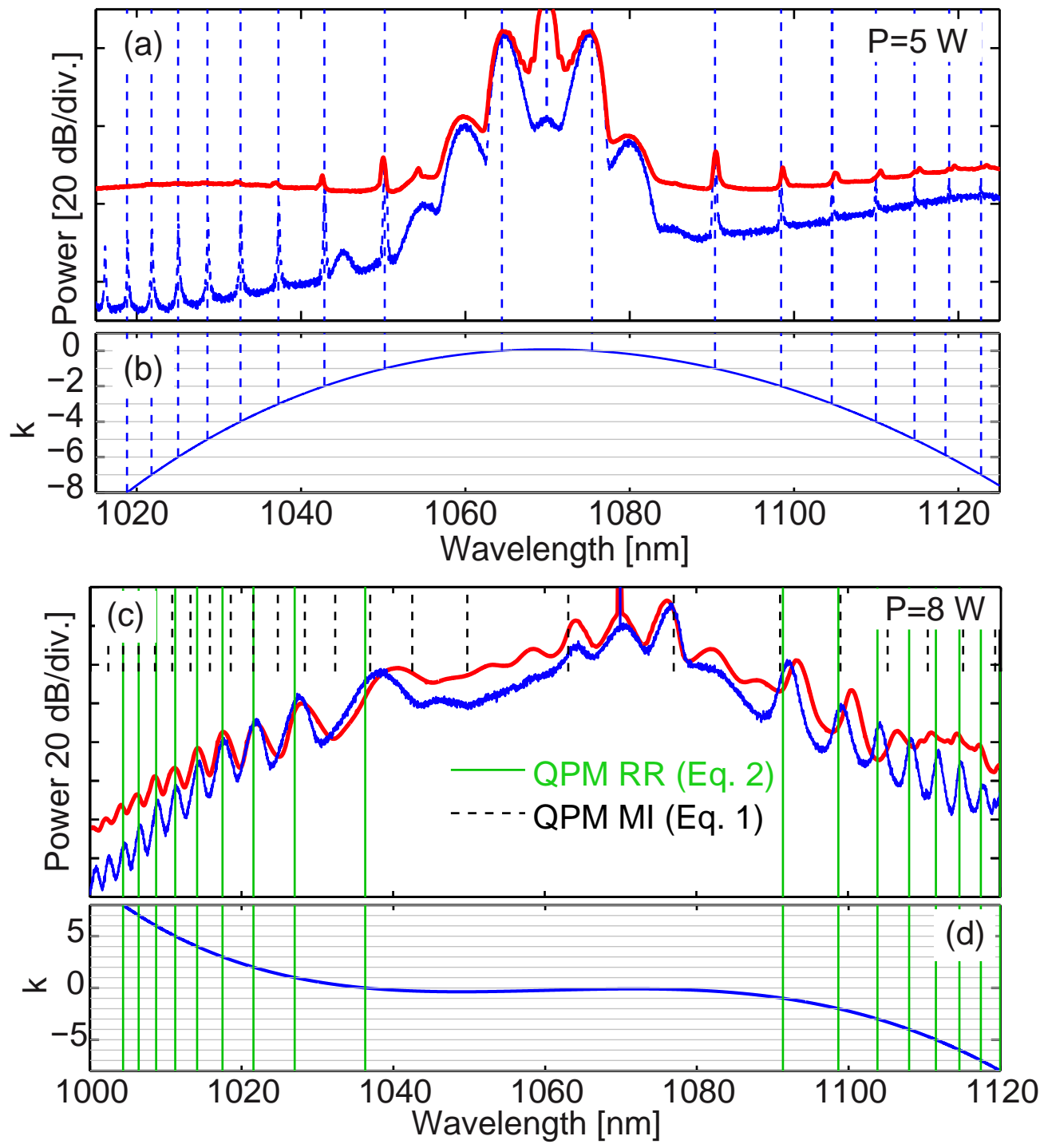

Fig. 20. Experimental (red curve) and numerical (blue curve) output spectra for (a) $5 \mathrm{~W}$ of pump power and (c) $8 \mathrm{~W}$ of pump power. (b) QPM relation. Vertical black lines correspond to the MI side lobe positions determined by the graphical solutions of Eq. (26) and (d) QPM relation for a soliton. The vertical green lines correspond to the RR positions determined by the graphical solution of Eq. (2) of Ref. [57] while black dashed lines to the one of Eq. (26). Adapted from [57] [IEEE Photonics Technology Letters, 28, 740 (2016)]. 
for MI side lobes (Eq. (26)). The fact that odd dispersion terms appear in this relation implies that resonant radiations are not symmetric from the pump. This makes an important difference with MI side bands. As can be seen in Fig. 20 (b), the positions of these resonant radiations are very well predicted from the theoretical model (vertical green lines from Eq. 27). For comparison, solutions of QPM MI (Eq. (26)) are superimposed in dashed black lines in this figure and it is crystal clear that this relation cannot predict the positions of the side bands in the spectrum depicted in Fig. 20 (b), except for the two first side lobes that correspond to conventional MI $(m=0)$. We can thus conclude that this spectrum is composed of two standard symmetric MI side lobes (position predicted with (Eq. (26), see dashed black lines in Fig. 20 (b)) in addition with many resonant radiations (position predicted with (Eq. (27), see solid green lines in Fig. 20 (b)). All the experimental results (red curve in Fig. 20 (b)) are confirmed by numerical simulations (blue curve in Fig. 20 (b)).

These experimental and numerical investigations demonstrate that the propagation of a continuous wave in the average anomalous dispersion region of a DOFs lead to the generation of a train of solitonic pulses, which are perturbed by periodic variations of the dispersion and shed energy to multiple resonant radiations. These radiations are not symmetric from the pump and their positions are very well predicted by a quasi phase matching relation involving odd dispersion terms (Eq. 27). A similar scenario occurs when a CW field propagates in the anomalous dispersion region of an uniform fiber [13, 14, 105]. One major difference is that quasi-phase matching relations for MI process and for resonant radiation emission must be considered to predict the side band positions in the spectrum.

We point out that multiple sidebands generated according to a type of dispersion relationship as in Eq. (27) can be also driven, in the normal GVD regime, by a pulse that undergoes wavebreaking thus forming a localized shock front. Such type of phenomenon have been experimentally observed in Ref. [103], and explained in terms of a unified perturbation theory which holds valid regardless of the solitonic or front nature of the localized wave that radiates. The quasi-momentum given by the periodicity, i.e. the RHS in Eq. (27), strongly enhances the resonant radiation induced by a shock wave, compared with the case of uniform fibers [106-108]. This is a general principle that find, for instance, a manifestation also in the context of multimode fibers [109].

Finally, we would like to mention that the impact of periodic dispersion has been investigated theoretically on temporal structures associated with the nonlinear regime of MI such as Kuznetsov-Ma breathers. It is shown that it strongly modifies their dynamics $[110,111]$, but up to know no experimental demonstration have been reported.

\section{PASSIVE CAVITIES MADE OF DISPERSION OSCILLATING FIBERS}

MI can occur in single pass fibers [6] but also in passive cavities thanks to the constructive interference between the external driving and re-circulating pulses [112-115]. The cavity detuning can be viewed as an additional degree of freedom to the system that must be accounted for in the phase matching relation that characterizes these systems [113]. We proposed to investigate passive cavities in which a DOF or GVD management is inserted. The additional periodicity introduced by the periodic variation of the dispersion drastically 

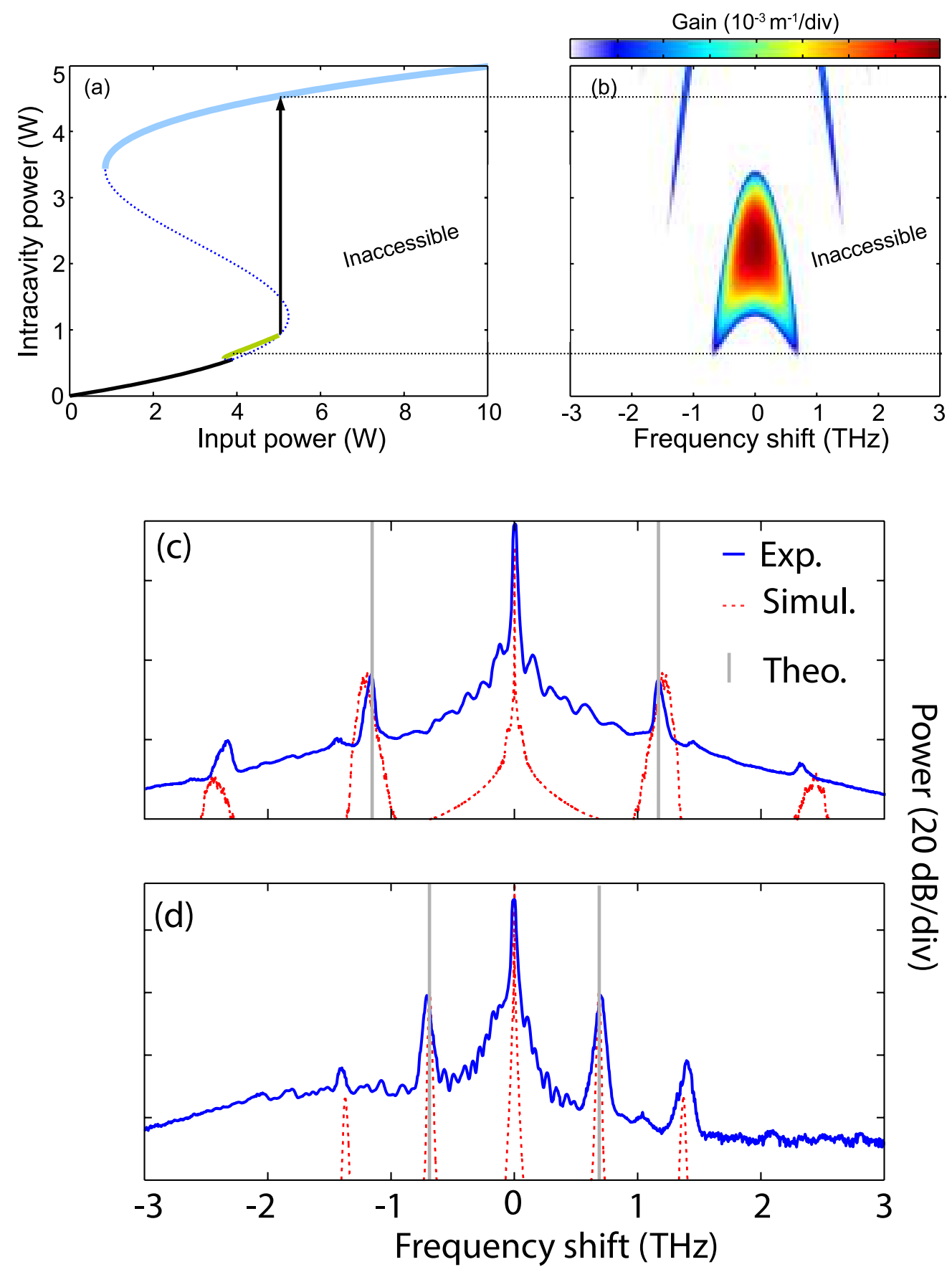

Fig. 21. (a) Bistable response of the cavity for the operating normalized detuning $\Delta=\delta / \alpha=4(\delta=\pi / 4.5 \mathrm{rad}$; loss coefficient $\alpha=0.157)$; the hatched region is unstable (unaccessible). (b) Pseudo-color level plot of the gain spectrum as a function of the intracavity power calculated from Floquet analysis [116]. (c-d) Comparison of experimental spectra (solid blue), spectra obtained from numerical integrations of the periodic LLE (dashed red), and analytical estimates (vertical line). (c) $P_{i n}=3.16 \mathrm{~W}$; (d) $P_{\text {in }}=1.97 \mathrm{~W}$. Adapted from [77] [Physical Review Letters, 116, 143901 (2016)]. 
modifies the dynamics of the system. Consequently, it motivated many theoretical and experimental investigations [77, 116-120]. Based on a modified version of the Lugiato Lefever equation (LLE) [121] including longitudinal variations of the dispersion [116], it can be shown theoretically via the Floquet analysis that these passive fiber ring cavities made of DOFs exhibit novel interesting features. We show that instabilities arising from two different origins can coexist in these systems: Turing instabilities as the ones observed in uniform passive fiber cavities, and Faraday instabilities, due to the periodic variation of the dispersion that act as a spatial periodic forcing term [77]. In order to clearly highlight the coexistence of these instabilities in the same physical system, we built a suitable cavity with dispersion varying periodically over the round-trip and operating with a normal average dispersion value [77]. We focused our attention on the bistable configuration since it provides the most striking illustration of the role played by the periodic variation of the dispersion. In particular the cavity that we built [77] is characterized by a piecewise constant dispersion profile with a total length of $51.6 \mathrm{~m}$, and is composed by a $50 \mathrm{~m}$ long dispersion shifted fiber $\left(\beta_{2}=2 p s^{2} / \mathrm{km}\right)$ directly spliced to a standard 90/10 coupler made of SMF-28 $\left(\beta_{2}=-19 p s^{2} / \mathrm{km}\right)$. The contribution of the variation of the nonlinear coefficient, that is slightly different for each fiber, has been verified to be negligible. Figure 21 (a) represents the bistable response of this cavity and the evolution of the gain spectrum as a function of the intra-cavity power is shown in a 2D plot in Fig. 21 (b). For input powers below $3.7 \mathrm{~W}$ we do not observe any spectral signature of periodic structures in the output spectrum. Indeed the system is stable and simply follows the lower branch of the steady-state response s(Fig. 21(a)). After reaching $3.7 \mathrm{~W}$ (the cavity threshold) the cavity is unstable (see green portion on the lower branch of Fig. 21 (a)) and leads to the appearance of 2 symmetric side bands at $0.7 \mathrm{THz}$ (Fig. 21(c)). These instabilities usually refers to as Turing instabilities since they do not originate from periodic variations of the dispersion but from the cavity itself [122, 123]. Then, when the power exceeds the value where the Turing branch merges on the stationary response, the Turing instability induces up-switching towards the upper branch. This branch presents narrowband Faraday instability (Fig. 21(b)) and hence two sidebands are still observed in the spectra (Fig. 21(c)), though at much larger frequency $(1.16 \mathrm{THz})$. These side bands originate from the periodic variation of the dispersion which act as a periodic forcing term. As can be seen, experimental spectra (blue curves) in Fig. 21 (c) are in excellent agreement with numerical simulations (dashed red curves) and with analytical predictions [77, 116] (vertical grey lines, $0.69 \mathrm{THz}$ and $1.15 \mathrm{THz}$, respectively). The large difference of frequency between the sidebands of Turing and Faraday types is the distinctive trait which allows us to unequivocally identify the different regimes of instability in our experiments. In these works the pump power was adiabatically increased. In order to capture the roundtrip-to-roundtrip dynamics of this modulated passive cavity, a dispersive Fourier technique has been used in Ref. [118]. These recording revealed a complex dynamics during which the system switches from one instability mode to the other without any evidence for their temporary coexistence. Furthermore, recent measurements have demonstrated that the Faraday mechanism, when the cavity is operating with weak dispersion, i.e. close to average zero dispersion wavelength, can be responsible for the generation of a huge number of sidebands [119].

These results demonstrate the feasibility of controlling the dynamics of a 
bistable system via periodic modulations and report the first observation of Turing and Faraday instabilities in the same system. They induced a renewed interest for the physics of passive cavities, and opened new fundamental questions on parametric resonances, period doubled temporal patterns that may arise in these dissipative systems [117, 124, 125]. Moreover, the results have contributed to extend the validity of the LLE to passive cavities with non-constant parameters [116]. A recent analysis reveals the limitation of such model compared with the original Ikeda-like map, while discussing the parametric origin of the different instability bands in the cavity system [117].

\section{CONCLUSION}

In this review we summarize recent progresses on modulation instability process in dispersion oscillating fibers in single pass configurations or in passive cavities. We provide a short qualitative description of MI in these waveguides, where we show that MI in DOFs can be relatively well described by a simple quasi-phase matching relation. This simplified approach allows to get a very good prediction of the position of multiple MI sidebands generated in these fibers. Then, a rigorous theoretical investigation of MI in these waveguides based the Floquet analysis is detailed. It shows that QPM predictions can be derived from this study and remains valid as long as the pump power remains relatively weak. However, no simple expression of the parametric gain is derived. To circumvent this problem, we obtain a simple analytical relation of the gain of each sideband from the truncated three wave model that allows to get a simple physical insight of the physics of the process. While approximated, this simple expression can be very useful for designing DOFs and to get a rapid understanding of the dynamics of MI in these waveguides. We then summarized main experimental results obtained in these fibers from the first experimental observation in microstructured fibers reported by some of us in 2014 . We then show that it can be observed in DOFs with different modulation formats ranging from sinusoidal to delta Dirac series, or to an optimized modulation allowing the achievement of a wide and flat gain band $(12 \mathrm{THz})$ that might be useful for short pulse amplification. All these investigations are performed in the linear regime of MI and we show that a more complex dynamics is achieved in the nonlinear regime of the process. Namely, a mix between QPM sidebands, harmonics or dispersive wave can occur under strong pumping conditions. Moreover, theoretical investigations reveal that Fermi Pasta Ulam recurrence is observable in DOFs while pumping in the normal dispersion region of DOFs and that strong parametric amplification outside the Floquet gain band is expected. Finally, a passive cavity made of a DOF is investigated theoretically and experimentally. It is shown that Turing instabilities as well as Faraday instabilities can be observed in the same physical system for the first time to our knowledge, and that one can switch from one instability regime to another by simply tuning the pump power. Finally, these investigations confirm that optical fibers are a fantastic experimental test beds to perform fundamental investigations in nonlinear optics.

\section{ACKNOWLEDGEMENTS}

The authors would like to thank all people involved in this work. 


\section{FUNDING INFORMATION}

This work was partly supported by the Agence Nationale de la Recherche through the ANR TOPWAVE, NoAWE, HEAFISY projects, the Labex CEMPI and Equipex FLUX through the "Programme Investissements d'Avenir", by the French Ministry of Higher Education and Research, the Nord-Pas de Calais Regional Council and Fonds Européen de Développement Économique Régional (FEDER) through the "Contrat de Projets État Région (CPER) 2007-2013", the HEAFISY project and the "Campus Intelligence Ambiante (CIA)".S.T. acknowledges kind hospitality and support from PhLAM and partial support from grant PRIN 2012BFNWZ2.

\section{REFERENCES}

1. V. E. Zakharov and L. A. Ostrovsky, "Modulation instability: The beginning," Physica D: Nonlinear Phenomena 238, 540-548 (2009).

2. T. Taniuti and H. Washimi, "Self-Trapping and Instability of Hydromagnetic Waves Along the Magnetic Field in a Cold Plasma," Phys. Rev. Lett. 21, 209-212 (1968).

3. T. B. Benjamin and J. E. Feir, "The Disintegration of Wave Trains on Deep Water Part 1. Theory," Journal of Fluid Mechanics 27, 417-430 (1967).

4. F. K. Abdullaev, B. B. Baizakov, S. A. Darmanyan, V. V. Konotop, and M. Salerno, "Nonlinear excitations in arrays of Bose-Einstein condensates," Physical Review A 64, 043606 (2001).

5. R. Lai and A. J. Sievers, "Modulational instability of nonlinear spin waves in easy-axis antiferromagnetic chains," Phys. Rev. B 57, 3433-3443 (1998).

6. K. Tai, A. Hasegawa, and A. Tomita, "Observation of modulational instability in optical fibers," Phys. Rev. Lett. 56, 135 (1986).

7. G. Agrawal, Nonlinear Fiber Optics, Fifth Edition (Academic Press, Amsterdam, 2012), 5th ed.

8. G. Van Simaeys, P. Emplit, and M. Haelterman, "Experimental demonstration of the fermi-pasta-ulam recurrence in a modulationally unstable optical wave," Phys. Rev. Lett. 87, 033902 (2001).

9. G. Van Simaeys, P. Emplit, and M. Haelterman, "Experimental study of the reversible behavior of modulational instability in optical fibers," J. Opt. Soc. Am. B 19, 477-486 (2002).

10. A. Mussot, A. Kudlinski, M. Droques, P. Szriftgiser, and N. Akhmediev, "Fermi-Pasta-Ulam Recurrence in Nonlinear Fiber Optics: The Role of Reversible and Irreversible Losses," Physical Review X 4, 011054 (2014). WOS:000333584500003.

11. B. Kibler, J. Fatome, C. Finot, G. Millot, F. Dias, G. Genty, N. Akhmediev, and J. M. Dudley, "The Peregrine soliton in nonlinear fibre optics," Nat Phys 6, 790-795 (2010).

12. B. Kibler, J. Fatome, C. Finot, G. Millot, G. Genty, B. Wetzel, N. Akhmediev, F. Dias, and J. M. Dudley, "Observation of Kuznetsov-Ma soliton dynamics in optical fibre," Scientific Reports 2 (2012).

13. J. M. Dudley, G. Genty, and S. Coen, "Supercontinuum generation in photonic crystal fiber," Rev. Mod. Phys. 78, 1135 (2006).

14. J. M. Dudley and J. Taylor, Supercontinuum Generation in Optical Fibers (Cambridge University Press, 2010), 1st ed.

15. D. R. Solli, C. Ropers, P. Koonath, and B. Jalali, "Optical rogue waves," Nature 450, 1054-1057 (2007).

16. N. Akhmediev, B. Kibler, F. Baronio, M. Belić, W.-P. Zhong, Y. Zhang, Wonkeun Chang, J. M. Soto-Crespo, P. Vouzas, P. Grelu, C. Lecaplain, K. Hammani, S. Rica, A Picozzi, M. Tlidi, K. Panajotov, A. Mussot, A. Bendahmane, Pascal Szriftgiser, G. Genty, J. Dudley, A. Kudlinski, A. Demircan, U. Morgner, Shalva Amiraranashvili, C. Bree, G. Steinmeyer, C. Masoller, N. G. R. Broderick, A. F. J. Runge, Miro Erkintalo, S. Residori, U. Bortolozzo, F. T. Arecchi, S. Wabnitz, C. G. Tiofack, S. Coulibaly, and M. Taki, "Roadmap on optical rogue waves and extreme events," J. Opt. 18, 063001 (2016).

17. P. Suret, R. E. Koussaifi, A. Tikan, C. Evain, S. Randoux, C. Szwaj, and S. Bielawski, "Single-shot observation of optical rogue waves in integrable turbulence using time microscopy," Nature Communications 7 , 13136 (2016).

18. B. Wetzel, D. Bongiovanni, M. Kues, Y. Hu, Z. Chen, S. Trillo, J. M. Dudley, S. Wabnitz, and R. Morandotti, "Experimental Generation of Riemann Waves in Optics: A Route to Shock Wave Control," Phys. Rev. Lett. 117, 073902 (2016).

19. R. Tang, J. Lasri, P. S. Devgan, V. Grigoryan, P. Kumar, and M. Vasilyev, "Gain characteristics of a frequency nondegenerate phase-sensitive fiber-optic parametric amplifier with phase self-stabilized input," Opt. Express 13, 10483-10493 (2005).

20. M. E. Marhic, Fiber Optical Parametric Amplifiers, Oscillators and Related Devices (Cambridge University Press, 2007), 1st ed.

21. J. Hansryd, P. A. Andrekson, M. Westlund, J. Li, and P. O. Hedekvist, "Fiber-based optical parametric amplifiers and their applications," IEEE Journal of Selected Topics in Quantum Electronics 8, 506-520 (2002).

22. C. Caucheteur, D. Bigourd, E. Hugonnot, P. Szriftgiser, A. Kudlinski, M. Gonzalez-Herraez, and A. Mussot, "Experimental demonstration of optical parametric chirped pulse amplification in optical fiber," Optics Letters 35, 1786-1788 (2010). 
23. A. Hasegawa, "Generation of a train of soliton pulses by induced modulational instability in optical fibers," Opt. Lett. 9, 288-290 (1984).

24. S. Radic, "Parametric Signal Processing," IEEE Journal of Selected Topics in Quantum Electronics 18, 670-680 (2012).

25. K. Tajima, "Compensation of soliton broadening in nonlinear optical fibers with loss," Opt. Lett. 12, 54-56 (1987).

26. V. A. Bogatyrev, M. M. Bubnov, E. M. Dianov, A. S. Kurkov, P. V. Mamyshev, A. M. Prokhorov, S. D. Rumyantsev, V. A. Semenov, S. L. Semenov, A. A. Sysoliatin, S. V. Chernikov, A. N. Gur'yanov, G. G. Devyatykh, and S. I. Miroshnichenko, "A single-mode fiber with chromatic dispersion varying along the length," Journal of Lightwave Technology 9 (1991).

27. P. V. Mamyshev, S. V. Chernikov, and E. M. Dianov, "Generation of fundamental soliton trains for high-bitrate optical fiber communication lines," IEEE Journal of Quantum Electronics 27 (1991).

28. S. V. Chernikov and P. V. Mamyshev, "Femtosecond soliton propagation in fibers with slowly decreasing dispersion," J. Opt. Soc. Am. B, JOSAB 8, 1633-1641 (1991).

29. A. Kudlinski, A. K. George, J. C. Knight, J. C. Travers, A. B. Rulkov, S. V. Popov, and J. R. Taylor, "Zerodispersion wavelength decreasing photonic crystal fibers for ultraviolet-extended supercontinuum generation," Opt. Exp. 14, 5715-5722 (2006).

30. A. Kudlinski, M. Lelek, B. Barviau, L. Audry, and A. Mussot, "Efficient blue conversion from a $1064 \mathrm{~nm}$ microchip laser in long photonic crystal fiber tapers for fluorescence microscopy," 18, 16640-16645.

31. E. Yablonovitch, T. J. Gmitter, and K. M. Leung, "Photonic band structure: The face-centered-cubic case employing nonspherical atoms," Phys. Rev. Lett. 67, 2295-2298 (1991).

32. C. Denz, S. Flach, and Y. S. Kivshar, eds., Nonlinearities in Periodic Structures and Metamaterials, vol. 150 of Springer Series in Optical Sciences (Springer Berlin Heidelberg, Berlin, Heidelberg, 2010).

33. N. J. Smith, F. M. Knox, N. J. Doran, K. J. Blow, and I. Bennion, "Enhanced power solitons in optical fibres with periodic dispersion management," Electronics Letters 32, 54-55 (1996).

34. I. R. Gabitov and S. K. Turitsyn, "Averaged pulse dynamics in a cascaded transmission system with passive dispersion compensation," Opt. Lett. 21, 327-329 (1996).

35. B. A. Malomed, Soliton Management in Periodic Systems (Springer, 2005), 1st ed

36. S. K. Turitsyn, B. G. Bale, and M. P. Fedoruk, "Dispersion-managed solitons in fibre systems and lasers," Physics Reports 521, 135 - 203 (2012). Dispersion-Managed Solitons in Fibre Systems and Lasers.

37. F. Matera, A. Mecozzi, M. Romagnoli, and M. Settembre, "Sideband instability induced by periodic power variation in long-distance fiber links," Opt. Lett. 18, 1499-1501 (1993).

38. N. J. Smith and N. J. Doran, "Modulational instabilities in fibers with periodic dispersion management," Opt. Lett. 21, 570-572 (1996).

39. J. C. Bronski and J. Nathan Kutz, "Modulational stability of plane waves in nonreturn-to-zero communications systems with dispersion management," Opt. Lett. 21, 937-939 (1996).

40. F. Consolandi, C. De Angelis, A.-D. Capobianco, G. Nalesso, and A. Tonello, "Parametric gain in fiber systems with periodic dispersion management," Optics Communications 208, 309-320 (2002).

41. A. Kumar, A. Labruyere, and P. Tchofo Dinda, "Modulational instability in fiber systems with periodic loss compensation and dispersion management," Optics Communications 219, 221-232 (2003).

42. F. K. Abdullaev, S. A. Darmanyan, S. Bischoff, and M. P. Sorensen, "Modulational instability of electromagnetic waves in media with varying nonlinearity," J. Opt. Soc. Am. B 14, 27-33 (1997).

43. F. K. Abdullaev and J. Garnier, "Modulational instability of electromagnetic waves in birefringent fibers with periodic and random dispersion," Phys. Rev. E 60, 1042 (1999).

44. S. Ambomo, C. M. Ngabireng, P. T. Dinda, A. Labruyère, K. Porsezian, and B. Kalithasan, "Critical behavior with dramatic enhancement of modulational instability gain in fiber systems with periodic variation dispersion," J. Opt. Soc. Am. B 25, 425-433 (2008).

45. M. Droques, A. Kudlinski, G. Bouwmans, G. Martinelli, and A. Mussot, "Dynamics of the modulation instability spectrum in optical fibers with oscillating dispersion," Phys. Rev. A 87, 013813 (2013).

46. A. Armaroli and F. Biancalana, "Tunable modulational instability sidebands via parametric resonance in periodically tapered optical fibers," Opt. Express 20, 25096-25110 (2012).

47. A. Armaroli and F. Biancalana, "Vector modulational instability induced by parametric resonance in periodically tapered highly birefringent optical fibers," Phys. Rev. A 87, 063848 (2013).

48. A. Armaroli and F. Biancalana, "Suppression and splitting of modulational instability sidebands in periodically tapered optical fibers because of fourth-order dispersion," Opt. Lett. 39, 4804-4807 (2014).

49. M. Droques, A. Kudlinski, G. Bouwmans, G. Martinelli, and A. Mussot, "Experimental demonstration of modulation instability in an optical fiber with a periodic dispersion landscape," Optics Letters 37, 4832 (2012).

50. M. Droques, A. Kudlinski, G. Bouwmans, G. Martinelli, A. Mussot, A. Armaroli, and F. Biancalana, "Fourthorder dispersion mediated modulation instability in dispersion oscillating fibers," Opt. Lett. 38, 3464-3467 (2013).

51. S. R. Nodari, M. Conforti, G. Dujardin, A. Kudlinski, A. Mussot, S. Trillo, and S. De Bièvre, "Modulational instability in dispersion-kicked optical fibers," Phys. Rev. A 92, 013810 (2015).

52. M. Conforti, A. Mussot, A. Kudlinski, S. Rota Nodari, G. Dujardin, S. De Biévre, A. Armaroli, and S. Trillo, "Heteroclinic Structure of Parametric Resonance in the Nonlinear Schrodinger Equation," Phys. Rev. Lett. 117, 013901 (2016).

53. F. Copie, A. Kudlinski, M. Conforti, G. Martinelli, and A. Mussot, "Modulation instability in amplitude modulated dispersion oscillating fibers," Optics Express 23, 3869-3875 (2015). WOS:000350872700004.

54. X. Wang, D. Bigourd, A. Kudlinski, K. K. Y. Wong, M. Douay, L. Bigot, A. Lerouge, Y. Quiquempois, and 
A. Mussot, "Correlation between multiple modulation instability side lobes in dispersion oscillating fiber," Optics Letters 39, 1881 (2014).

55. C. Finot, F. Feng, Y. Chembo, and S. Wabnitz, "Gain sideband splitting in dispersion oscillating fibers," Optical Fiber Technology 20, 513-519 (2014).

56. F. Feng, P. Morin, Y. K. Chembo, A. Sysoliatin, S. Wabnitz, and C. Finot, "Experimental demonstration of spectral sideband splitting in strongly dispersion oscillating fibers," Opt. Lett. 40, 455-458 (2015).

57. M. Conforti, S. Trillo, A. Kudlinski, and A. Mussot, "Multiple QPM Resonant Radiations Induced by MI in Dispersion Oscillating Fibers," IEEE Photonics Technology Letters 28, 740-743 (2016).

58. C. Finot and S. Wabnitz, "Influence of the pump shape on the modulation instability process induced in a dispersion-oscillating fiber," J. Opt. Soc. Am. B, JOSAB 32, 892-899 (2015).

59. C. Finot, A. Sysoliatin, and S. Wabnitz, "Nonlinear parametric resonances in quasiperiodic dispersion oscillating fibers," Optics Communications 348, 24-30 (2015).

60. C. Finot, J. Fatome, A. Sysoliatin, A. Kosolapov, and S. Wabnitz, "Competing four-wave mixing processes in dispersion oscillating telecom fiber," Opt. Lett. 38, 5361-5364 (2013).

61. K. Kikuchi, C. Lorattanasane, F. Futami, and S. Kaneko, "Observation of quasi-phase matched four-wave mixing assisted by periodic power variation in a long-distance optical amplifier chain," Photonics Technology Letters, IEEE 7, 1378-1380 (1995).

62. S. G. Murdoch, M. D. Thomson, R. Leonhardt, and J. D. Harvey, "Quasi-phase-matched modulation instability in birefringent fibers," Opt. Lett. 22, 682-684 (1997).

63. S. G. Murdoch, R. Leonhardt, J. D. Harvey, and T. A. B. Kennedy, "Quasi-phase matching in an optical fiber with periodic birefringence," J. Opt. Soc. Am. B 14, 1816-1822 (1997).

64. J. B. Driscoll, N. Ophir, R. R. Grote, J. I. Dadap, N. C. Panoiu, K. Bergman, and R. M. Osgood, "Widthmodulation of Si photonic wires for quasi-phase-matching of four-wave-mixing: experimental and theoretical demonstration," Optics Express 20, 9227 (2012).

65. M. E. Marhic and F. S. Yang, "High-Nonlinearity Fiber Optical Parametric Amplifier with Periodic Dispersion Compensation," J. Lightwave Technol. 17, 210 (1999).

66. A. S. Y. Hsieh, G. K. L. Wong, S. G. Murdoch, S. Coen, F. Vanholsbeeck, R. Leonhardt, and J. D. Harvey, "Combined effect of Raman and parametric gain on single-pump parametric amplifiers," Opt. Express 15, 8104-8114 (2007).

67. L. Provost, C. Moreau, G. Melin, X. Rejeaunier, L. Gasca, P. Sillard, and P. Sansonetti, "Dispersionmanaged fiber with low chromatic dispersion slope," in "OFC 2003 Optical Fiber Communications Conference, 2003.", (2003), pp. 151-153 vol.1.

68. A. A. Sysoliatin, A. K. Senatorov, A. I. Konyukhov, L. A. Melnikov, and V. A. Stasyuk, "Soliton fission management by dispersion oscillating fiber," Optics Express 15, 16302-16307 (2007).

69. P. Russell, "Photonic Crystal Fibers," Science 299, 358-362 (2003).

70. T. A. Birks, J. C. Knight, and P. S. Russell, "Endlessly single-mode photonic crystal fiber," Opt. Lett. 22, 961-963 (1997).

71. K. Saitoh and M. Koshiba, "Empirical relations for simple design of photonic crystal fibers," Opt. Express 13, 267-274 (2005).

72. F. K. Abdullaev, S. A. Darmanyan, A. Kobyakov, and F. Lederer, "Modulational instability in optical fibers with variable dispersion," Physics Letters A 220, 213-218 (1996).

73. S. Trillo and S. Wabnitz, "Dynamics of the nonlinear modulational instability in optical fibers," Opt. Lett. 16, 986-988 (1991).

74. K. Inoue and T. Mukai, "Signal wavelength dependence of gain saturation in a fiber optical parametric amplifier," Opt. Lett. 26, 10-12 (2001).

75. G. Cappellini and S. Trillo, "Third-order three-wave mixing in single-mode fibers: exact solutions and spatial instability effects," J. Opt. Soc. Am. B, JOSAB 8, 824-838 (1991).

76. S. G. Murdoch, R. Leonhardt, and J. D. Harvey, "Polarization modulation instability in weakly birefringent fibers," Opt. Lett. 20, 866-868 (1995).

77. F. Copie, M. Conforti, A. Kudlinski, A. Mussot, and S. Trillo, "Competing turing and faraday instabilities in longitudinally modulated passive resonators," Phys. Rev. Lett. 116, 143901 (2016).

78. L. G. Wright, Z. Liu, D. A. Nolan, M.-J. Li, D. N. Christodoulides, and F. W. Wise, "Self-organized instability in graded-index multimode fibres," Nature Photonics 10, nphoton.2016.227 (2016).

79. N. Christensen, R. Leonhardt, and J. D. Harvey, "Noise characteristics of cross-phase modulation instability light," Optics Communications 101, 205-212 (1993).

80. B. Wetzel, A. Stefani, L. Larger, P. A. Lacourt, J. M. Merolla, T. Sylvestre, A. Kudlinski, A. Mussot, G. Genty, F. Dias, and J. M. Dudley, "Real-time full bandwidth measurement of spectral noise in supercontinuum generation," Scientific Reports 2, 882 (2012). WOS:000311890700001.

81. T. Godin, B. Wetzel, T. Sylvestre, L. Larger, A. Kudlinski, A. Mussot, A. Ben Salem, M. Zghal, G. Genty, F. Dias, and J. M. Dudley, "Real time noise and wavelength correlations in octave-spanning supercontinuum generation," Opt. Express 21, 18452-18460 (2013).

82. S. R., H. G., and J. B. R. C., "Fluctuations and correlations in modulation instability," Nat Photon 6, 463-468 (2012).

83. K. Goda and B. Jalali, "Dispersive Fourier transformation for fast continuous single-shot measurements," Nat Photon 7, 102-112 (2013).

84. S. B. Cavalcanti, J. C. Cressoni, H. R. da Cruz, and A. S. Gouveia-Neto, "Modulation instability in the region of minimum group-velocity dispersion of single-mode optical fibers via an extended nonlinear Schrödinger equation," Phys. Rev. A 43, 6162 (1991). 
85. S. B. Cavalcanti, J. C. Cressoni, H. R. da Cruz, and A. S. Gouveia-Neto, "Modulation instability in the region of minimum group-velocity dispersion of single-mode optical fibers via an extended nonlinear Schrödinger equation," Physical Review A 43, 6162 (1991).

86. F. K. Abdullaev, S. A. Darmanyan, S. Bischoff, P. L. Christiansen, and M. P. Sørensen, "Modulational instability in optical fibers near the zero dispersion point," Optics Communications 108, 60-64 (1994).

87. S. Pitois and G. Millot, "Experimental observation of a new modulational instability spectral window induced by fourth-order dispersion in a normally dispersive single-mode optical fiber," Optics Communications 226, 415-422 (2003).

88. J. D. Harvey, R. Leonhardt, S. Coen, G. K. L. Wong, J. Knight, W. J. Wadsworth, and P. St.J. Russell, "Scalar modulation instability in the normal dispersion regime by use of a photonic crystal fiber," Opt. Lett. 28, 2225-2227 (2003).

89. S. Atkins, A. Rosen, A. Bekker, and B. Fischer, "Evolution of localization in frequency for modulated light pulses in a recirculating fiber loop," Optics Letters 28, 2228-2230 (2003).

90. B. V. Chirikov, "A universal instability of many-dimensional oscillator systems," Physics Reports 52, 263-379 (1979).

91. B. Fischer, A. Rosen, and S. Fishman, "Localization in frequency for periodically kicked light propagation in a dispersive single-mode fiber," Opt. Lett. 24, 1463-1465 (1999).

92. M. Karlsson, "Four-wave mixing in fibers with randomly varying zero-dispersion wavelength," Journal of the Optical Society of America B 15, 2269-2275 (1998).

93. M. Farahmand and M. de Sterke, "Parametric amplification in presence of dispersion fluctuations," Optics Express 12, 136-142 (2004).

94. B. Sévigny, O. Vanvincq, C. Valentin, N. Chen, Y. Quiquempois, and G. Bouwmans, "Four-wave mixing stability in hybrid photonic crystal fibers with two zero-dispersion wavelengths," Optics Express 21, 3085930873 (2013).

95. B. P.-P. Kuo, J. M. Fini, L. Grüner-Nielsen, and S. Radic, "Dispersion-stabilized highly-nonlinear fiber for wideband parametric mixer synthesis," Optics Express 20, 18611-18619 (2012).

96. A. Mussot, A. Kudlinski, R. Habert, I. Dahman, G. Mélin, L. Galkovsky, A. Fleureau, S. Lempereur, L. Lago, D. Bigourd, T. Sylvestre, M. W. Lee, and E. Hugonnot, "20 THz-bandwidth continuous-wave fiber optical parametric amplifier operating at $1 \mu \mathrm{m}$ using a dispersion-stabilized photonic crystal fiber," Optics Express 20, 28906-28911 (2012).

97. C. Fourcade-Dutin, Q. Bassery, D. Bigourd, A. Bendahmane, A. Kudlinski, M. Douay, and A. Mussot, "12 thz flat gain fiber optical parametric amplifiers with dispersion varying fibers," Opt. Express, OE 23, 10103-10110 (2015).

98. M. E. Fermann and I. Hartl, "Ultrafast fibre lasers," Nat Photon 7, 868-874 (2013).

99. F. Feng, J. Fatome, A. Sysoliatin, Y. Chembo, S. Wabnitz, and C. Finot, "Wavelength conversion and temporal compression of pulse train using dispersion oscillating fibre," Electronics Letters 50, 768-770 (2014).

100. N. N. Akhmediev and V. I. Korneev, "Modulation instability and periodic solutions of the nonlinear schrödinger equation," Teor. Mat. Fiz. 69, 189-194 (1986).

101. S. Trillo and S. Wabnitz, "Nonlinear modulation of coupled waves in birefringent optical fibers," Physics Letters A 159, 252 - 256 (1991).

102. C. De Angelis, M. Santagiustina, and S. Trillo, "Four-photon homoclinic instabilities in nonlinear highly birefringent media," Phys. Rev. A 51, 774-791 (1995).

103. M. Conforti, S. Trillo, A. Mussot, and A. Kudlinski, "Parametric excitation of multiple resonant radiations from localized wavepackets," Scientific Reports 5, 9433 (2015).

104. M. Conforti and S. Trillo, "Dispersive wave emission from wave breaking," Opt. Lett. 38, 3815-3818 (2013).

105. A. Mussot, E. Lantz, H. Maillotte, T. Sylvestre, C. Finot, and S. Pitois, "Spectral broadening of a partially coherent CW laser beam in single-mode optical fibers," Opt. Express 12, 2838-2843 (2004).

106. M. Conforti and S. Trillo, "Dispersive wave emission from wave breaking," Opt. Lett. 38, 3815-3818 (2013).

107. K. E. Webb, Y. Q. Xu, M. Erkintalo, and S. G. Murdoch, "Generalized dispersive wave emission in nonlinear fiber optics," Opt. Lett. 38, 151-153 (2013).

108. M. Conforti, F. Baronio, and S. Trillo, "Resonant radiation shed by dispersive shock waves," Phys. Rev. A 89, 013807 (2014).

109. L. G. Wright, S. Wabnitz, D. N. Christodoulides, and F. W. Wise, "Ultrabroadband dispersive radiation by spatiotemporal oscillation of multimode waves," Phys. Rev. Lett. 115, 223902 (2015).

110. U. Al Khawaja, S. M. Al-Marzoug, H. Bahlouli, and F. K. Abdullaev, "Modulational instability of the Kuznetsov-Ma breather in optical fibers with constant and periodic dispersion," Communications in Nonlinear Science and Numerical Simulation 32, 1-9 (2016)

111. C. G. L. Tiofack, S. Coulibaly, M. Taki, S. De Bièvre, and G. Dujardin, "Periodic modulations controlling Kuznetsov-Ma soliton formation in nonlinear Schrödinger equations," Physics Letters A 381, 1999-2003 (2017).

112. M. Haelterman, S. Trillo, and S. Wabnitz, "Dissipative modulation instability in a nonlinear dispersive ring cavity," Optics Communications 91, 401-407 (1992).

113. M. Haelterman, S. Trillo, and S. Wabnitz, "Additive-modulation-instability ring laser in the normal dispersion regime of a fiber," Opt. Lett. 17, 745-747 (1992).

114. S. Coen and M. Haelterman, "Modulational Instability Induced by Cavity Boundary Conditions in a Normally Dispersive Optical Fiber," Phys. Rev. Lett. 79, 4139-4142 (1997).

115. S. Coen and M. Haelterman, "Continuous-wave ultrahigh-repetition-rate pulse-train generation through modulational instability in a passive fiber cavity," Opt. Lett. 26, 39-41 (2001) 
116. M. Conforti, A. Mussot, A. Kudlinski, and S. Trillo, "Modulational instability in dispersion oscillating fiber ring cavities," Optics Letters 39, 4200 (2014).

117. M. Conforti, F. Copie, A. Mussot, A. Kudlinski, and S. Trillo, "Parametric instabilities in modulated fiber ring cavities," Opt. Lett. 41, 5027-5030 (2016).

118. F. Copie, M. Conforti, A. Kudlinski, S. Trillo, and A. Mussot, "Dynamics of turing and faraday instabilities in a longitudinally modulated fiber-ring cavity," Opt. Lett. 42, 435-438 (2017).

119. F. Copie, M. Conforti, A. Kudlinski, S. Trillo, and A. Mussot, "Modulation instability in the weak dispersion regime of a dispersion modulated passive fiber-ring cavity," Opt. Express 25, 11283-11296 (2017).

120. F. Copie, M. Conforti, A. Kudlinski, A. Mussot, F. Biancalana, and S. Trillo, "Instabilities in passive dispersion oscillating fiber ring cavities," The European Physical Journal D 71, 133 (2017).

121. L. A. Lugiato and R. Lefever, "Spatial Dissipative Structures in Passive Optical Systems," Phys. Rev. Lett. 58, 2209-2211 (1987).

122. S. Coen, M. Haelterman, P. Emplit, L. Delage, L. M. Simohamed, and F. Reynaud, "Bistable switching induced by modulational instability in a normally dispersive all-fibre ring cavity," J. Opt. B: Quantum Semiclass. Opt. 1, 36 (1999).

123. S. Coen and M. Haelterman, "Competition between modulational instability and switching in optical bistability," Optics Letters 24, 80 (1999).

124. A. M. Perego, N. Tarasov, D. V. Churkin, S. K. Turitsyn, and K. Staliunas, "Pattern generation by dissipative parametric instability," Phys. Rev. Lett. 116, 028701 (2016).

125. K. Staliunas, C. Hang, and V. V. Konotop, "Parametric patterns in optical fiber ring nonlinear resonators," Phys. Rev. A 88, 023846 (2013).

\section{AUTHOR BIOGRAPHIES}

Arnaud Mussot received the Ph.D. in Physics from University of Besancon in 2004. Until 2005 he had a post doc position at the French Atomic Agency (CEA) in Bordeaux in France. In 2005 he joined the University of Lille as a lecturer and he was appointed full professor in 2014. Since 2015, he is junior member of the Institut Universitaire de France (IUF). His interests focus on nonlinear fiber optics. He has co-authored more than 110 papers in peer-review journals.

Matteo Conforti received the MS in Electronic Engineering and the Ph.D. degree in Electronic Instrumentation form the University of Brescia in 2003 and 2007, respectively. From 2014 he is CNRS researcher at the Laboratoire de Physique des Lasers, Atomes et Molécules (PhLAM) in Lille. His main research interests are Nonlinear Optics, Solitons, Rogue and Shock Waves. He has co-authored arround 100 papers in peer-review journals. 
Stefano Trillo received the MS in Electronic Engineeging, 1982, and Ph.D. in Applied Electromagnetism, 1987, both from University of Rome La Sapienza. Until 1998 he was employed as senior scientist at Fondazione Ugo Bordoni in Rome. Since 1998 he joined the University of Ferrara, where he is now full professor and director of a $\mathrm{PhD}$ program. His interests span the fields of photonics, nonlinear optics, quantum electronics, electromagnetic propagation, modelling of optical devices, and nonlinear waves in general. In these areas his contributions are witnessed by more than 200 papers in high impact journals, several book chapters and a long list of conference papers. He is Fellow of the Optical Society of America.

François Copie graduated from the University of Lille, France where he received his MS degree in light-matter interactions in 2014. The same year he started a thesis on the "modulation instability process in dispersion oscillating cavities" at the University of Lille where he carried out research on parametric processes in topographic fibers and resonators.

Alexandre Kudlinski received the Ph.D. in Physics from University of Lille in 2005. Until 2006 he was employed as a research officer in the CPPM at University of Bath. In 2006 he joined the University of Lille as a lecturer and he was appointed full professor in 2016. His interests focus on photonic crystal fibers and nonlinear fiber optics. He has co-authored more than 120 papers in peer-review journals. 